\title{
The UNC5C Netrin Receptor Regulates Dorsal Guidance of Mouse Hindbrain Axons
}

\author{
Doyeun Kim and Susan L. Ackerman \\ Howard Hughes Medical Institute and The Jackson Laboratory, Bar Harbor, Maine 04609
}

The cerebellum receives its input from multiple precerebellar nuclei located in the brainstem and sends processed information to other brain structures via the deep cerebellar neurons. Guidance molecules that regulate the complex migrations of precerebellar neurons and the initial guidance of their leading processes have been identified. However, the molecules necessary for dorsal guidance of precerebellar axons to the developing cerebellum or for guidance of decussating axons of the deep nuclei are not known. To determine whether Unc $5 c$ plays a role in the dorsal guidance of precerebellar and deep cerebellar axons, we studied axonal trajectories of these neurons in $U n c 5 c^{-1-}$ mice. Our results show that $U n c 5 c$ is expressed broadly in the precerebellar and deep cerebellar neurons. Unc5c deletion disrupted long-range dorsal guidance of inferior olivary and pontine axons after crossing the midline. In addition, dorsal guidance of the axons from the medial deep cerebellar and external cuneate neurons was affected in Unc5c $c^{-1-}$ mice, as were anterior migrations of pontine neurons. Coincident with the guidance defects of their axons, degeneration of neurons in the external cuneate nucleus and subdivisions of the inferior olivary nucleus was observed in $U n c 5 c^{-1-}$ mice. Lastly, transgenic expression of Unc5c in deep neurons and pontine neurons by the Atoh1 promoter rescued defects of the medial deep cerebellar and pontine axons observed in Unc5c $c^{-1-}$ embryos, demonstrating that $U n c 5 c$ acts cell autonomously in the guidance of these axons. Our results suggest that Unc5c plays a broad role in dorsal guidance of axons in the developing hindbrain.

\section{Introduction}

The cerebellum processes information received from precerebellar neurons in the brainstem and conveys this information to other brain areas. The circuitry of the cerebellar system is developed through complicated steps of neuronal and axonal migrations. Precerebellar axons, as well as some soma, migrate ventrally and cross midline. These axons continue moving dorsally to project to contralateral cerebellum (Altman and Bayer, 1987a,b,c; Sotelo, 2004). Within the cerebellum, the direction of axonal projections of the deep cerebellar neurons (DCNs) correlates with their future position. Axons from lateral/intermediate deep neurons extend ventrally out of the cerebellum via the superior cerebellar peduncle (SCP) and project to target neurons in the thalamus. In contrast, axons from medial deep neurons migrate dorsally, crossing the cerebellar midline via the hook bundle to project to their targets in the brainstem (Altman and Bayer, 1985).

Molecular determinants of some of these complex events in vivo have been demonstrated using knock-out mice. Both netrin-1 and its receptor DCC and members of the Slit/Robo

Received 0ct. 5, 2010; revised Dec. 4, 2010; accepted Dec. 11, 2010.

This research was supported by National Institute for Neurological Disease and Stroke Grant NS35900 to S.L.A. and CORE Grant CA34196 to The Jackson Laboratory. We thank Dr. Jane Johnson for the Atoh1 promoter cassette and the Atoh1-tauGFP mice, Drs. Robert Burgess and Thomas Gridley for comments on the manuscript, the Microinjection Core at The Jackson Laboratory for generating transgenic mice, and Jesse Hammer for graphics assistance. S.L.A. is an investigator of the Howard Hughes Medical Institute.

Correspondence should be addressed to Dr. Susan L. Ackerman, The Jackson Laboratory, 600 Main Street, Bar Harbor, ME 04609. E-mail: susan.ackerman@jax.org.

DOI:10.1523/JNEUROSCI.5254-10.2011

Copyright $\odot 2011$ the authors $\quad 0270-6474 / 11 / 312167-13 \$ 15.00 / 0$ guidance system have been shown to regulate the ventral guidance and midline crossing of precerebellar neurons and their axons (Bloch-Gallego et al., 1999; Causeret et al., 2002; Marillat et al., 2004; Di Meglio et al., 2008; Marcos et al., 2009). Similarly, ventral guidance and/or crossing of migrating axons of lateral/ intermediate deep neurons have been shown to be under RIG-1/ ROBO1/2 control (Tamada et al., 2008).

In contrast to our understanding of ventral axon guidance mechanisms in the nervous system, the molecular cues responsible for dorsally directed axon guidance, including that of the precerebellar and hook bundle axons, are largely unknown. Like attraction, dorsal repulsion has been suggested to be driven by cues derived from the floor plate (Serafini et al., 1996; Shirasaki et al., 1996). In addition, studies in invertebrates suggest that different mechanisms exist for dorsal repulsion of axons depending on the distance of the growth cones from the floor plate (Keleman and Dickson, 2001; MacNeil et al., 2009). By extension to vertebrates, many hindbrain axons require long-range repulsion from the floor plate, depending on their axonal trajectories and the location of their soma. In contrast, distinct molecular mechanisms might govern the short-range repulsion of spinal commissural axons from the floor plate (Long et al., 2004; Jaworski et al., 2010).

$U n c 5 c$ is a repulsive receptor for netrin, a bifunctional axon guidance cue (Hong et al., 1999). Here, we demonstrate that Unc $5 c$ is expressed widely in precerebellar and cerebellar neurons and is necessary for dorsal guidance of their axons. First, we demonstrate that $U n c 5 c$ is required for the long-range dorsal guidance of axons of the inferior olivary (IO) neurons and pontine neurons after crossing midline. We also show that axons of $U n c 5 c^{-1-}$ 
external cuneate neurons fail to navigate the dorsal turn that is necessary for their projection to the cerebellum. Further, Unc5c disruption leads to migration defects of the pontine neurons. Lastly, we demonstrate that $U n c 5 c$ is required for the initial dorsal guidance of the hook bundle. Together, our results demonstrate that $U n c 5 c$ plays a major role in hindbrain development via its function in dorsal axonal guidance.

\section{Materials and Methods}

Mice. C57BL/6J (B6);SJL Unc5c $c^{-1-}$ mice were generated by backcrossing F1 $U n c 5 c^{+/-}$mice obtained from a B6.Unc5c $c^{+/-} \times \mathrm{SJL} / \mathrm{J}$ mating to B6.Unc5c $c^{+/-}$mice. These mice were used for all experiments involving adult mice, and both male and female mice were used. B6.Unc5c ${ }^{-1-}$ mice were used for all experiments involving embryos or neonates [the latter designated as postnatal day $0(\mathrm{P} 0)]$ and were obtained from heterozygous matings. Embryos were generated by timed matings, and the day on which a vaginal plug was detected was designated as embryonic day 0.5

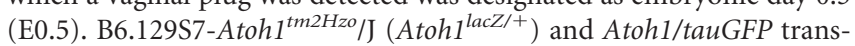
genic mice (TgAtoh1-tauGFP) were obtained from The Jackson Laboratory and Dr. Jane Johnson (University of Texas Southwestern Medical Center, Dallas, TX), respectively (Imondi et al., 2007).

To express Unc5c in Atoh1-expressing neurons, a transgenic construct was made by subcloning the mouse Unc5c cDNA into the Atoh1 promoter cassette containing the $1.7 \mathrm{~kb}$ Atohl enhancer (Helms et al., 2000). The first 46 aa of UNC5C, including the signal peptide, were replaced by a Myc tag and an immunoglobulin Ig $\kappa$ B leader sequence. The construct was microinjected into the pronuclei of B6 embryos and founders were identified by PCR using 5' -CCTCGTAGACAGCCTTGCTCG-3' and 5' GCTCAGGTGGGTCAGATGGA-3' primers that are complimentary to the Atoh 1 promoter and the $U n c 5 c$ coding sequence, respectively. When necessary, B6.TgAtoh1-Unc5c;Unc5c ${ }^{+/-}$mice were crossed to F1 B6;SJL$U n c 5 c^{+1-}$ mice to generate viable TgAtoh1-Unc5c;Unc5c $c^{-1-}$ mice. All other experiments involving Atoh1-Unc5c transgenic mice were done on an inbred B6 background. The Animal Care and Use Committee of The Jackson Laboratory approved all animal protocols.

Histology. Adult mice were perfused transcardially with Bouin's fixative and decapitated, and heads were immersion fixed in the same fixative overnight. Brains were dissected out, processed, and embedded in paraffin. Sections $(7 \mu \mathrm{m})$ were cut from paraffin-embedded brains and stained with cresyl violet and/or Luxol fast blue.

In situ hybridization. In situ hybridization was performed as described previously (Welch et al., 2004). Briefly, embryonic heads (or brains, if older than E14.5) were dissected, embedded in OCT (Tissue-Tek), and frozen using liquid nitrogen. Tissues were cryosectioned at $15 \mu \mathrm{m}$ and mounted onto SuperFrost Plus slides (Fisher Scientific). Frozen sections were fixed with $4 \%$ paraformaldehyde (PFA), acetylated, bleached, and hybridized with digoxigenin (DIG)-labeled $U n c 5 c$ riboprobes at $68^{\circ} \mathrm{C}$ overnight. Riboprobes corresponding to the $U n c 5 c$ cDNA were labeled using a DIG RNA labeling kit (Roche) and diluted 1:1000 in buffer for hybridization (Ackerman et al., 1997). Riboprobes were detected using an anti-DIG-POD fragment (Roche) followed by tyramide signal amplification (TSA)-plus procedures (PerkinElmer). After optimal staining was obtained $(\sim 15 \mathrm{~min})$, sections were washed, counterstained with Hoechst dye $(1 \mu \mathrm{g} / \mathrm{ml})$, and mounted using Fluoromount-G (Southern Biotech). Images were taken with a fluorescent microscope (Olympus) equipped with the Retiga 2000R camera (QImaging).

Immunohistochemistry. Embryonic heads or dissected embryonic/ neonatal brains (if older than E16.5) were fixed in $4 \%$ PFA, at $4{ }^{\circ} \mathrm{C}$ overnight. Adult mice were transcardially perfused with $4 \%$ PFA, and brains were dissected before immersion fixation. Tissues were processed, paraffin embedded, and sectioned at $7 \mu \mathrm{m}$ thickness. After deparaffinization, antigen retrieval was performed by boiling sections for $5 \mathrm{~min}$ in sodium citrate solution $(0.01 \mathrm{M}, \mathrm{pH} 6)$. Sections were incubated at $4{ }^{\circ} \mathrm{C}$ overnight with primary antibodies including rabbit anti-UNC5C (1:300, generated using the $\mathrm{C}$ terminus 400 aa of recombinant mouse protein as immunogen), mouse anti-BRN3A (1:50, Santa Cruz Biotechnology), mouse anticalbindin D-28k (1:200, Sigma), rabbit anti-calretinin (1:400, Millipore Bioscience Research Reagents), rabbit anti-RIG1 (1:500, Sigma), rabbit
anti-TBR1 (1:800, Millipore Bioscience Research Reagents), rabbit anticleaved caspase 3 (1:200, Cell Signaling Technology), guinea pig antivGLUT2 (1:400, Millipore Bioscience Research Reagents), mouse antiMYC (1:500, Zymed), and mouse $2 \mathrm{H} 3$ or 3A10 (1:200, supernatant, Developmental Studies Hybridoma Bank, University of Iowa, Iowa City, IA) in PBST (0.3\% Triton X-100 in PBS) containing 5\% donkey serum. For BARHL1 immunostaining, frozen sections were made as described above. Sections were postfixed with 4\% PFA for $5 \mathrm{~min}$ and washed and incubated with rabbit anti-BARHL1 (1:200, Sigma) antibodies at $4{ }^{\circ} \mathrm{C}$ overnight. After washing, sections were incubated with $\mathrm{Cy} 3$-conjugated donkey anti-rabbit IgG (1:200; Jackson ImmunoResearch Laboratories) or Alexa Fluor 488-conjugated donkey anti-mouse IgG (1:200; Invitrogen) antibodies at room temperature (RT) for $1 \mathrm{~h}$. For 3,3'-diaminobenzidine detection of bound neurofilament and calbindin antibodies, sections were incubated with biotin-conjugated anti-mouse IgG (1:100, Sigma) antibody and ExtrAvidin-peroxidase (1:50, Sigma) at RT for $1 \mathrm{~h}$ each. After washing in PBST, sections were counterstained and imaged as described above.

Cell counting. To count embryonic medial deep neurons and inferior olivary neurons, serial sections $(7 \mu \mathrm{m})$ in the coronal plane were collected from E16.5 paraffin-embedded wild-type and $U n c 5 \mathrm{c}^{-1-}$ hindbrains. Sections were immunostained with anti-TBR1 or anti-BRN3A antibodies to visualize medial deep neurons or inferior olivary neurons, respectively. TBR1-positive neurons in the cerebellum and BRN3Apositive neurons in the inferior olivary nucleus (ION) were counted in 5 sections spaced every 100 and $112 \mu \mathrm{m}$ through the medial deep nucleus and ION, respectively. Inferior olivary neurons were counted in adult mice in a similar manner, except that calbindin-positive neurons were counted in sections spaced every $200 \mu \mathrm{m}$ through the ION. To count developing external cuneate nucleus (ECN) neurons, coronal serial frozen sections $(15 \mu \mathrm{m})$ were collected and immunostained with anti-BARHL1 antibodies. Immunoreactive cells in the ECN region (dorsolateral medulla) were counted in four sections spaced every $180 \mu \mathrm{m}$. Cells were counted using the ImageJ cell counter program (National Institutes of Health, Bethesda, $\mathrm{MD}$ ), and $t$ tests were performed to statistically analyze results. For all experiments, three brains per each genotype were used.

$X$-Gal staining. Wholemount X-Gal (5-bromo-4-chloro-3-indolyl- $\beta$ D-galactopyranoside) staining was performed as described previously (Wang et al., 2005). In brief, E16.5 embryonic brains were dissected out and fixed by immersion in $4 \%$ PFA for $20 \mathrm{~min}$. After washing with PBS, brains were incubated at $37^{\circ} \mathrm{C}$ in $\mathrm{X}$-Gal staining solution overnight. Wholemount images were taken under the dissecting microscope (Nikon) equipped with Spot camera (Diagnostic Instruments).

Neurotracer labeling. DiI (1,1' -dioctadecyl 3,3,3',3'-tetramethylindocarbocyanine) labeling was performed as described by Bloch-Gallego et al. (1999). In brief, E16.5 embryos or P0 neonates were transcardially perfused with $4 \%$ PFA. A cerebellar hemisphere was carefully exposed and 4-5 crystals of DiI (Invitrogen D282) were implanted. Tissues were incubated in $4 \% \mathrm{PFA}$ at $37^{\circ} \mathrm{C}$ in the dark for 4 weeks for distal labeling of the ECN/lateral reticular nucleus (LRN) and the ION and 1 week for proximal labeling of the hook bundle and the SCP. After incubation, brains were dissected, embedded in $4 \%$ low melt agarose (Bio-Rad), and vibratome sectioned $(120 \mu \mathrm{m})$. Sections were mounted on the glass slide and coverslipped using PBS containing $20 \mu \mathrm{g} / \mathrm{ml}$ Hoechst dye. For direct placement of DiI into the ION or ECN, perfused neonatal heads were carefully dissected at the neck to expose the ventral or dorsal medulla. A crystal of DiI was implanted in the medulla at midline caudal to the basilar-vertebral artery branch for the ION or in the dorsolateral region just under the caudal cerebellum for the ECN. Tissues were incubated for $2 \mathrm{~d}$ before vibratome sectioning. For direct labeling of the pontine nucleus (PN), E16.5 embryos were perfused with 4\% PFA and brains were dissected. DiI was placed into the PN that was visualized in some embryos using GFP expression from the Atoh1-tauGFP transgene. To visualize the normal and ectopic position of the PN in $U n c 5 c^{-1-}$ embryos, GFP expression from the Atoh1-tauGFP transgene was always used as a guide. Tissues were incubated for $5 \mathrm{~d}$, and DiI-labeled axons were observed in wholemount brains. Images were taken under the fluorescent dissecting microscope using a Cy3 filter set (Nikon) equipped with Spot 
camera (Diagnostic Instruments). For all DiI labeling experiments, at least three mice were used per each genotype.

Fluorogold labeling procedures followed previously described methods with small modifications (Yamada et al., 2007). In brief, mice were anesthetized by an intraperitoneal injection of $2 \%$ tribromoethanol. Incisions were made in the skin overlaying the cerebellum and two small holes were made in one side of the skull over the declive in the vermis and the crus I lobule of the hemisphere. Fluorogold solution $(0.2 \mu \mathrm{l}, 4 \%$ in PBS; Fluorochrome) was injected at a depth of $2 \mathrm{~mm}$ using a $1 \mu \mathrm{l} \mathrm{Ham-}$ ilton syringe. The syringe was held vertically in place for $5 \mathrm{~min}$ to prevent leakage. After $3 \mathrm{~d}$, brains were dissected out and cryosectioned $(15 \mu \mathrm{m})$ as described above. Sections were postfixed with $4 \%$ PFA and immunostained with antibodies to calbindin D-28k before mounting with Fluoromount-G. For olivocerebellar topographic analysis, a single injection of Fluorogold was made in either crus I in the hemispheres or the declive in the vermis. Six cryosections spaced every $150 \mu \mathrm{m}$ were collected, and Fluorogold puncta were counted in each section using the ImageJ cell counter program (National Institutes of Health). Fluorogold labeling in P5 animals was done similarly with a single injection in crus I, and mice were killed $2 \mathrm{~d}$ later. Sections were counterstained with antibodies against BRN3A to visualize the ION. Images were taken with an Axiocam camera (Zeiss) using a wide band UV filter set 02 . For all experiments, three mice were analyzed per each genotype.

Fluorescein-conjugated dextran amine labeling was performed using the same injection procedure for Fluorogold with the following modifications. Two small holes were made in one side of the skull over the declive in the vermis and the sulcus paravermis to target the medial and intermediate deep cerebellar nuclei. Fluorescein-conjugated dextran amine solution $(0.2 \mu \mathrm{l}, 50 \mathrm{mg} / \mathrm{ml}$ in PBS, $10 \mathrm{kDa}$; Invitrogen D1820) was injected vertically into the cerebellum at a depth of $3 \mathrm{~mm}$. Two days later, mice were transcardially perfused with $4 \%$ PFA, and brains were dissected and vibratome sectioned. Images were taken under the fluorescent dissecting microscope using FITC filter set (Nikon) equipped with Spot camera (Diagnostic Instruments). Injection into the medial/intermediate deep nuclei was confirmed by the position of the needle mark and the location of the region of intense staining. Three mice were analyzed per each genotype.

\section{Results}

\section{Abnormalities in the cerebellar commissure and inferior cerebellar peduncle in the Unc5c $\mathrm{c}^{-/-}$hindbrain}

We have previously demonstrated that $U n c 5 c$ is necessary for granule cell and Purkinje cell migrations during cerebellar development (Ackerman et al., 1997; Przyborski et al., 1998). However, $U n c 5 c$ is strongly expressed in additional cerebellar and other hindbrain neuronal precursors, suggesting it may play further roles in hindbrain development. To investigate this possibility, we performed histological analysis of wild-type and $U n c 5 c^{-1-}$ hindbrains. We observed that the inferior cerebellar peduncle (ICP), the axonal tract by which medullary precerebellar neurons send information to the cerebellum, was absent in mutant mice (Fig. 1A,B). This finding was consistent with a previous report on the cerebellar vermis defect $(c v d)$ mutant rat that was later demonstrated to have a spontaneous mutation in the Unc5c gene (Kuwamura et al., 1997; Kuramoto et al., 2004). However, both the SCP, which projects from the cerebellum to the midbrain and pons, and the middle cerebellar peduncle, which is composed of mossy fibers projecting from the pontine nucleus to the cerebellum, were present in mutant mice (Fig. $1 A, B)$ (data not shown). In addition to ICP abnormalities, the cerebellar commissure, which conveys cerebellar output from deep neurons to several nuclei in the contralateral brainstem, including the vestibular nuclei and the LRN, was dramatically reduced in the mutant brain (Ito, 1984) (Fig. 1C,D). While commissural defects were apparent in the $U n c 5 c^{-1-}$ cerebellum, the size and medial/lateral position of the three deep nuclei appeared

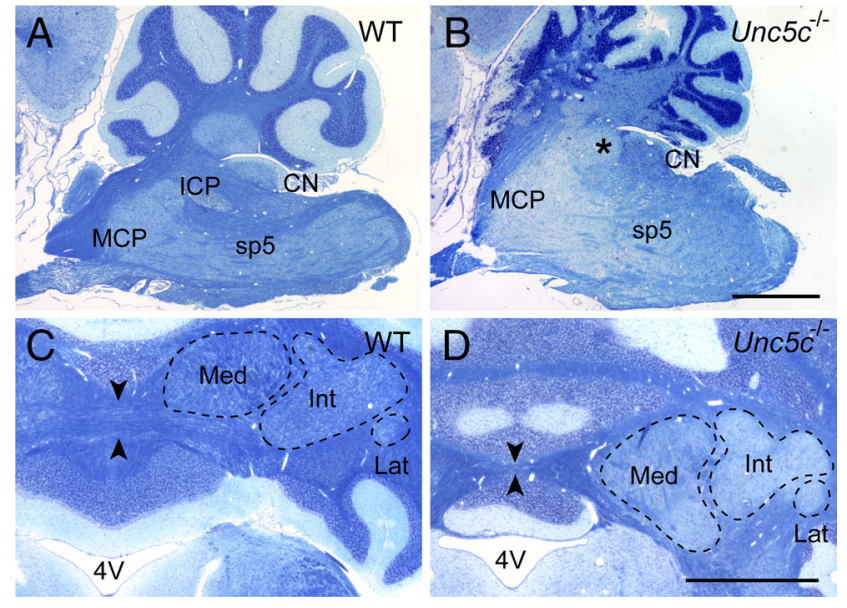

Figure 1. The ICP and the cerebellar commissure are defective in $U n C 5 C^{-1-}$ mice. $A-D$, Sagittal $(\boldsymbol{A}, \boldsymbol{B})$ or horizontal $(\boldsymbol{C}, \boldsymbol{D})$ sections of wild-type (WT) $(\boldsymbol{A}, \boldsymbol{C})$ or Unc5C $\mathrm{C}^{-1-}(\boldsymbol{B}, \boldsymbol{D})$ hindbrain were stained with Luxol fast blue to visualize axon tracts. Sections were counterstained with cresyl violet. Note the absence (indicated by an asterisk) of the ICP $(\boldsymbol{B})$ and reduction in the cerebellar commissure (flanked by arrowheads) (D) in the $U n \mathrm{CC}^{-1-}$ brain. The deep nuclei are outlined in dashed lines $(\boldsymbol{C}, \boldsymbol{D})$. CN, Cochlear nucleus; MCP, middle cerebellar peduncle; Med, medial deep cerebellar nucleus; Int, intermediate deep cerebellar nucleus; Lat, lateral deep cerebellar nucleus; 4V, fourth ventricle. Scale bars, $1 \mathrm{~mm}$.

grossly normal. However, these nuclei were slightly displaced rostrally, consistent with ectopic positioning of some granule cells and Purkinje cells in the midbrain (Fig. 1D) (Ackerman et al., 1997).

\section{Expression of $U n c 5 c$ in the developing medulla and cerebellum}

To begin to define the underlying sources of the ICP and cerebellar commissural defects in the Unc5c ${ }^{-1-}$ mouse, we analyzed the expression of Unc5c during embryonic hindbrain development. Deep cerebellar neuron progenitors transiently settle in the nuclear transitory zone (NTZ) after their generation from the upper rhombic lip. Expression of $U n c 5 c$ in the NTZ was apparent at E13.5 (Fig. 2A). In addition, high levels of expression were observed in Purkinje cell progenitors in the differentiation zone as previously reported (Przyborski et al., 1998).

Axons from medullary precerebellar nuclei (the ION, the ECN, and the LRN) project to the cerebellum via the ICP, and axons of this tract begin arriving in the developing cerebellum by E16/17 in rat (equivalent to E13/14 in mouse) (Wassef et al., 1992). In the E13.5 medulla, Unc5c expression was observed in medially located cells in the developing ION (data not shown). High expression was observed throughout the ION at E14.5, but expression decreased by E16.5 (Fig. 2B) (data not shown). We also detected Unc5c expression at E14.5 in the posterior extramural stream, located just under the pial membrane, which contains migrating ECN and LRN precursors (Fig. $2 B$ ). In addition, we detected Unc5c expression in pontine neurons at E16.5 (Fig. 2C). Like IO neurons, Unc5c expression was detected in the pontine neurons close to the midline but not in the migrating neurons found in the anterior extramural stream at E16.5.

To detect whether UNC5C was localized on developing axons, we generated rabbit polyclonal antibodies to the UNC5C intracellular region. Immunofluorescence of the E13.5 cerebellum demonstrated localization of UNC5C in the cell sparse area adjacent to the dorsal surface of the NTZ through which developing axons from deep cerebellar neurons horizontally project (Fig. $2 D$ ). In a coronal view of the cerebellum at E14.5, UNC5C immu- 
noreactivity was detected in horizontal fibers near the position of the presumptive hook bundle, the axons of the deep neurons that project across the cerebellar midline (Fig. 2E). We confirmed that these Unc5cexpressing axon fibers are from deep neurons by immunostaining adjacent sections with anti-TBR1 antibodies, a transcription factor expressed in all deep neurons at this developmental stage (Fig. 2 F) (Fink et al., 2006). In addition, UNC5C was also present on the presumptive early ICP fibers (Fig. $2 D, E)$. In the E13.5 medulla, UNC5C localization was observed in the marginally located circumferential tract, which includes the presumptive olivocerebellar tract (Fig. $2 G)$. UNC5C levels were also high in cells of the developing cochlear nucleus and vestibular ganglion at E13.5 (Fig. 2G). No signal was observed in the $U n c 5 c^{-1-}$ embryonic brain, confirming the specificity of the antibody (supplemental Fig. 1, available at www. jneurosci.org as supplemental material).

\section{Defects in projections of the}

precerebellar nuclei in $U n c 5 c^{-1-}$ mice

Climbing fibers from the ION cross mid-

line and then project dorsally along the

outer edge of the medulla toward the cerebellum. In contrast, the cuneocerebellar and reticulocerebellar tracts project ipsilaterally toward the cerebellum from the ECN and the LRN, joining the contralaterally projecting ION axons at dorsal and ventral positions, respectively (Altman and Bayer, 1997). To visualize the development of axonal projections from the precerebellar neurons to the cerebellum, we unilaterally inserted DiI into the P0 mutant and wild-type cerebellum. The olivocerebellar tract and the contralateral ION and the ipsilateral ECN and LRN were clearly labeled in wild-type brains (Fig. $3 A)(n=6)$. Although the ipsilateral LRN was also labeled in sections of mutant brainstem, we could not detect labeling of any neurons in the ECN or ION, or their axons that project along the lateral edge of the medulla (Fig. $3 B)(n=6)$.

Loss of Unc5c misroutes axonal projections but not migration of the ION precursors

The ION arises from the ventricular zone of the medulla. Between E12 and E14 these precursors tangentially migrate in the submarginal zone from the dorsal to the ventral medulla (de Diego et al., 2002). Many ION precursors fail to reach the ventral medulla in $\mathrm{Ntn1}^{-/-}$and $\mathrm{Dcc}{ }^{-1-}$ embryos, demonstrating that netrin plays a role in the attraction of ION progenitors to ventral midline (BlochGallego et al., 1999; Marcos et al., 2009).

To exclude the possibility that the failure of retrograde labeling of the $U n c 5 c^{-l-}$ ION comes from the malformation of this nucleus during development, we analyzed the structure of the ION in mutant and wild-type brains after IO neuronal migrations are complete. Brain sections from E16.5 mutant and wild-type embryos were immunostained with antibodies to the BRN3A transcription factor, which is highly expressed in the developing ION. Analysis of the medulla of $U n c 5 c^{-1-}$ embryos demonstrated that the ION appeared normal both in size and gross structure (Fig. $4 A, B)$. Furthermore, no significant differences in the number of BRN3A-positive cells were observed between the wild-type and the Unc5c $c^{-1-}$ ION (wild type, $1249 \pm 244$; mutant, $1336 \pm 188$ mean $\pm \mathrm{SD}, p=0.76 ; n=3$ for each genotype; see Materials and Methods for details).

To narrow the potential sites of guidance defects in $U n c 5 c^{-1-}$ IO axons, we asked whether mutant axons, like wild-type axons, cross midline. Coronal sections of the E13.5 medulla were immunostained with antibodies to RIG-1/ROBO3, which is highly localized in the growing inferior olivary, ECN, and LRN axons (Marillat et al., 2004). RIG-1 immunostaining in submarginal crossing fibers (i.e., the presumptive IO axons) at E13.5 and E14.5 (data not shown) was similar in the mutant and wild-type brain, suggesting that although $U n c 5 c^{-1-}$ axons fail to project correctly to the cerebellum, guidance of these axons across midline is similar to that of wild-type axons (Fig. 4C,D). In addition, no differences in RIG-1-positive LRN/ECN fibers that project more marginally in the medulla were observed between the wild-type and mutant brains. As expected, RIG-1-positive fibers at midline were greatly reduced in $\mathrm{Ntn} 1^{-1-}$ embryos in which IO and ECN/ LRN axons do not project across midline (data not shown) (Marcos et al., 2009).

To further define $U n c 5 c^{-1-}$ ION axon defects, we performed direct labeling of the P0 wild-type and mutant olivocerebellar tracts by placing DiI in the ION region. Unlike wild-type axons, which projected dorsally along the lateral aspect of medulla, mutant axons turned rostrally and then projected parallel to the midline through the ventral medulla (Fig. $4 E, F)(n=3$ for each genotype). To confirm that these abnormal axon trajectories observed in the mutant brain include IO axons, we performed immunohistochemistry with antibodies to calbindin D-28k, which is expressed on both neurons and axons of the ION on sections of wild-type and $U n c 5 c^{-1-}$ adult hindbrains. As visualized in coronal sections of the medulla, wild-type ION axons extended perpendicular to the rostral/caudal axis of the medulla, consistent with their dorsal trajectories from the ION (Fig. 4G). Interestingly, IO axons were present in the adult $U n c 5 c^{-1-}$ hindbrain, 

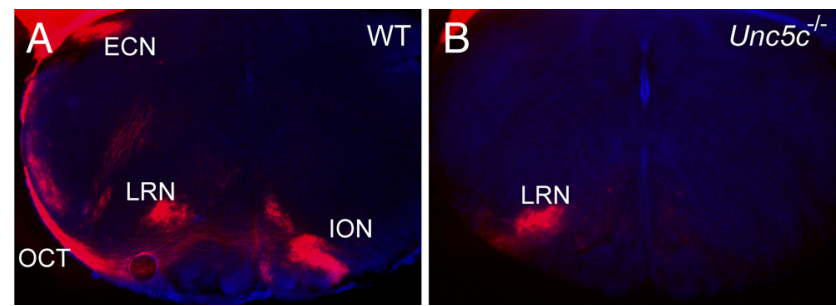

Figure 3. Axons of the ECN and the ION do not project to the cerebellum in Unc5C neonatal mice. $\boldsymbol{A}, \boldsymbol{B}$, Dil was placed unilaterally into cerebella of P0 wild-type (WT) $(\boldsymbol{A})$ and Unc5 $\mathrm{C}^{-1-}(\boldsymbol{B})$ mice, and hindbrains were coronally sectioned. The contralateral ION, the olivocerebellar tract $(0 C T)$, the ipsilateral ECN, and the LRN were labeled with Dil in the wild-type medulla, but only the LRN was labeled in the medulla of $U n \mathrm{CS}^{-1-}$ mice. Nuclei were counterstained with Hoechst dye.
WT
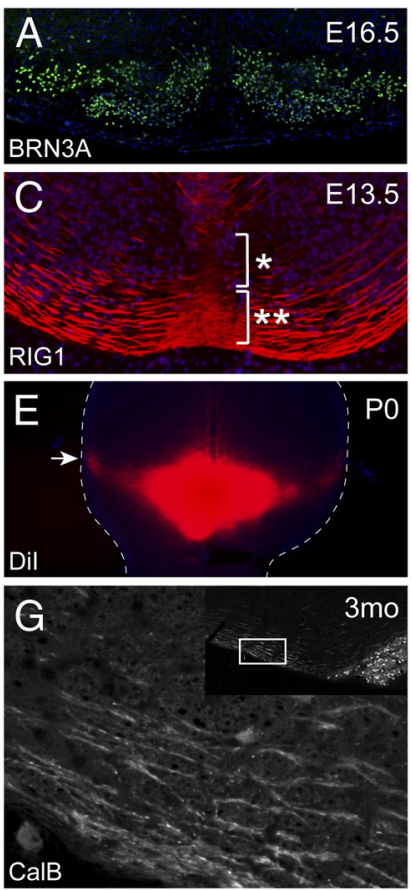
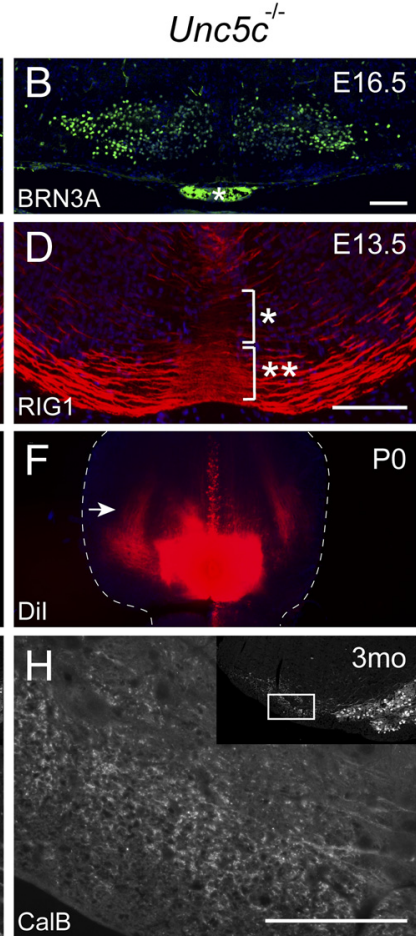

Figure 4. Unc5c is necessary for dorsal guidance of inferior olivary axons. $\boldsymbol{A}, \boldsymbol{B}$, Coronal sections from $\mathrm{E} 16.5$ wild-type (WT) and Unc $5 \mathrm{C}^{-1-}$ hindbrains were immunostained with antibodies to BRN3A to demonstrate that the neurons of the ION migrate properly in Unc $5 \mathrm{C}^{-1-}$ embryos. An asterisk ( $\boldsymbol{B})$ denotes blood vessel staining artifacts. $\boldsymbol{C}, \boldsymbol{D}$, Coronal sections of the E13.5 medulla from wild-type $(\boldsymbol{C})$ and mutant $(\boldsymbol{D})$ embryos were immunostained with anti-RIG1 antibodies to visualize 10 axons. Submarginally (I0) and marginally (ECN/LRN) migrating axons are indicated by a single asterisk and a double asterisk, respectively. Note that the relative intensity of RIG1 staining at ventral midline (brackets) does not differ between wild-type and Unc $5 \mathrm{C}^{-1-}$ embryos, suggesting that mutant axons cross midline. $\boldsymbol{E}, \boldsymbol{F}$, A single crystal of Dil was placed into the ION region of brains from $\mathrm{PO}$ wild-type $(\boldsymbol{E})$ and mutant $(\boldsymbol{F})$ mice. A horizontal section of the ventral brainstem is shown with the lateral edges of the brainstem outlined by dashed lines. Note that the olivocerebellar tract (arrows) projects laterally in the wild-type brain, whereas it turns rostrally in the mutant brain. $\mathbf{G}, \boldsymbol{H}$, Coronal sections of the adult medulla from 3-month-old (3mo) wild-type and mutant mice were immunostained with anti-calbindin D-28k (CalB) antibodies. Compare the longitudinal appearance of I0 axons in the wild-type brain with the punctate appearance of mutant axons. Lower-magnification images are shown in the insets to demonstrate the position (outlined by rectangles) of the corresponding highmagnification image. Scale bars, $10 \mu \mathrm{m}$.

suggesting that these misrouted axons might form functional synapses. However, in contrast to the calbindin-positive axons that project laterally in the wild-type medulla, punctate calbindin immunostaining was observed in cross-sectioned axons residing a short distance from midline in the $U n c 5 c^{-/-}$medulla (Fig. 4H). This staining pattern is consistent with the abnormal rostral projections of mutant axons observed in the neonatal hindbrain (Fig. $4 E, F)$. The abnormal longitudinal trajectory of mutant IO axons was confirmed in sagittal sections of hindbrain (supplemental Fig. 2, available at www.jneurosci.org as supplemental material). Examination of these sections demonstrated that IO axons continue to project rostrally from posterior medulla toward the pons where, at the level of the PN, they turn dorsally toward the cerebellum.

\section{The olivocerebellar tract is delayed in reaching the} cerebellum, and selective degeneration of IO neurons occurs in $U n c 5 c^{-1-}$ mice

The ION is the only source for climbing fibers that directly innervate specific zones of Purkinje cells along the medial/lateral axis (Altman and Bayer, 1997; Sotelo, 2004). Climbing fibers from the posterior ION primarily synapse on Purkinje cells located in the vermis, whereas climbing fibers of the anterior ION terminate on more laterally located Purkinje cells and comprise most of the synapses in the cerebellar hemispheres.

To determine whether climbing fibers contact the Purkinje cells in the mutant cerebellum, we visualized the terminals of these axons by immunohistochemistry with antibodies to vGLUT2, which is specifically expressed on varicosities of the climbing fibers at the climbing fiber/Purkinje cell dendrite synapse (Hioki et al., 2003). Examination of immunostained sections revealed clear localization of vGLUT2 on both wild-type and mutant Purkinje cell dendrites, although staining intensity was somewhat lower in the $U n c 5 c^{-1-}$ cerebellum, particularly in the hemispheres (supplemental Fig. 3, available at www.jneurosci.org as supplemental material). We also observed vGLUT2 staining on mislocalized Purkinje cells in the pons and midbrain (data not shown).

To confirm that IO axons eventually reach the cerebellum, we unilaterally injected the retrograde neurotracer Fluorogold into the cerebellum of adult wild-type and mutant mice. In both wildtype and mutant brains, labeling could be observed in all subdivisions of the contralateral, but not the ipsilateral ION (Fig. $5 A, B)$ (data not shown). To narrow the timing of cerebellar invasion of IO axons in $U n c 5 c^{-1-}$ mice, we performed unilateral Fluorogold injections in the cerebellum of P5 mice $(n=3$ for each genotype). The contralateral IONs of both wild-type and mutant mice were labeled at this time, indicating that mutant IO axons reach the cerebellum within $5 \mathrm{~d}$ after birth (Fig. $5 C, D$ ).

In addition to the abnormal trajectory of the mutant olivocerebellar tract and the corresponding delay of this tract in reaching the developing cerebellum, we consistently observed a reduction in the size of the ION in the adult medulla of mutant mice. Histological analysis of the mutant ION revealed that the number of neurons was dramatically reduced relative to the wild-type ION, particularly in the principal olive (PO) and dorsal accessory olive (DAO) (Fig. 5E,F) (Azizi and Woodward, 1987). Sections from the wild-type and $U n c 5 c^{-1-}$ medulla were immunostained with calbindin D-28k antibodies, and immunopositive neurons were quantified in each ION subdivision (Fig. 5G; supplemental Fig. 4, available at www.jneurosci.org as supplemental material) $(n=3$ for each genotype; see Materials and Methods). The total number of neurons in the mutant ION was $\sim 60 \%$ that of the wild-type ION. Interestingly, the decrease in neurons in the mutant ION displayed compartment specificity, with $\sim 70 \%$ reduction in the number of neurons in the $\mathrm{PO}$ and $\mathrm{DAO}$, and no apparent reduction in neurons within the medial accessory olive (MAO).

Climbing fiber/ Purkinje cell synapses initially form around E19 in the mouse (Mason et al., 1990). Our results suggest that failure of 

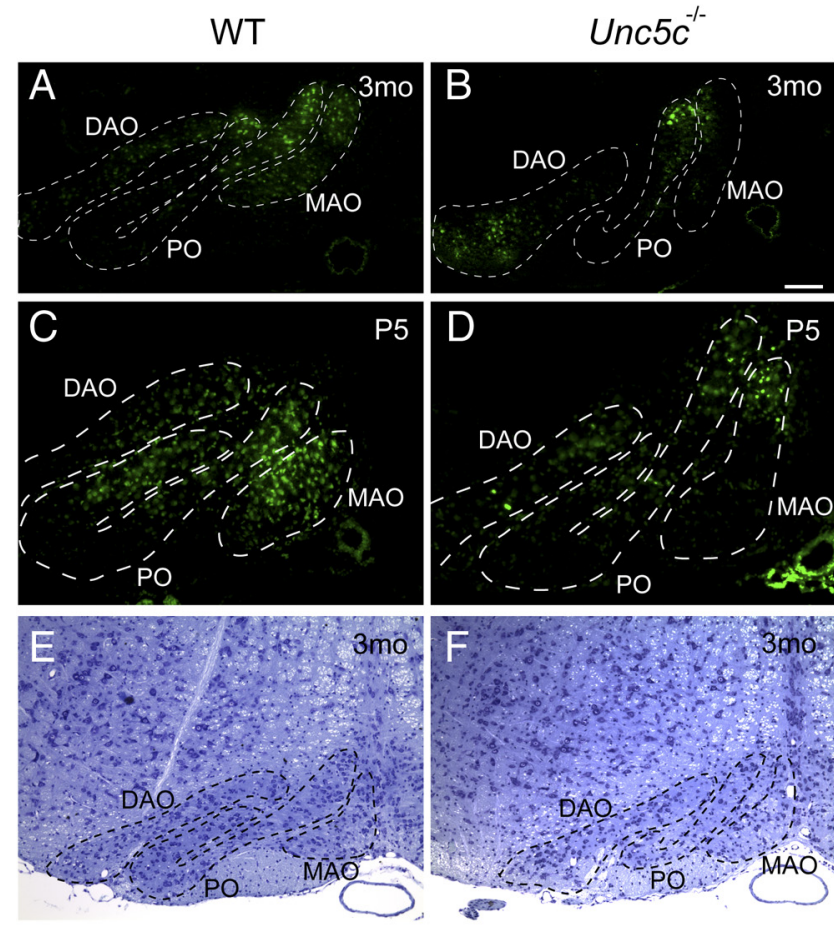

$\mathrm{G}$

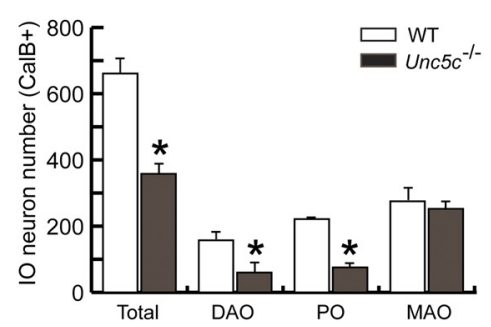

Figure 5. Delayed cerebellar innervation by 10 axons and selective degeneration of 10 neurons in the Unc $5 \mathrm{C}^{-1-}$ hindbrain. $\boldsymbol{A}-\boldsymbol{D}$, Fluorogold was injected unilaterally into the cerebellum of 3-month-old (3mo) $(\boldsymbol{A}, \boldsymbol{B})$ and postnatal day 5 (P5) (C, D) wild-type and mutant mice. Labeled neurons were observed in all subdivisions of the contralateral but not ipsilateral ION in both wild-type (WT) $(\boldsymbol{A}, \boldsymbol{C})$ and mutant $(\boldsymbol{B}, \boldsymbol{D})$ brains. Coronal sections of the medulla were immunostained with anti-calbindin D-28k antibodies (data not shown) to delineate the ION subdivisions (shown by dashed lines). $\boldsymbol{E}, \boldsymbol{F}$, Coronal sections of medulla from 3-month-old wild-type $(\boldsymbol{E})$ and $U n c 5 c^{-1-}(\boldsymbol{F})$ mice were stained with cresyl violet. The number of large diameter neurons in the $\mathrm{DA} 0$ and $\mathrm{PO}$, but not the $\mathrm{MAO}$, was dramatically reduced in the mutant. G, The medulla from 3-month-old wild-type and mutant adult mice were serially sectioned and immunostained with anti-calbindin D-28k antibodies. The number of immunoreactive cells in each subdivision of the ION was counted (see Materials and Methods). All values are mean \pm SD ${ }^{*} p<0.005$. Scale bar $(\boldsymbol{A}-\boldsymbol{F}), 10 \mu \mathrm{m}$.

climbing fibers to either reach their cerebellar targets during the late embryonic/early postnatal period and/or to form synapses with Purkinje cells may result in death of IO neurons. To test this hypothesis, we coimmunostained sections from P0 brains with antibodies to cleaved caspase- 3 and BRN3A (supplemental Fig. 5A-F, available at www.jneurosci.org as supplemental material). Cleaved caspase3positive neurons were detected in the mutant $\mathrm{PO}$, the $\mathrm{DAO}$, and the dorsal cap of $\mathrm{MAO}$, but not the wild-type $\mathrm{ION}$, and many positive neurons were also reactive with antibodies to BRN3A. This result demonstrates that many $U n c 5 c^{-1-}$ IO neurons undergo apoptosis at the time synapses normally form.

To determine whether loss of Unc5c alters the topography of climbing fiber projections, we unilaterally injected Fluorogold into the declive lobule of the vermis or crus I of the cerebellar hemisphere in wild-type and $U n c 5 c^{-1-}$ mice $(n=3$ for each genotype at each injection point). In both control and mutant animals, vermal injections preferentially labeled the contralateral posterior ION (supplemental Fig. 6, available at www.jneurosci. org as supplemental material). In contrast, preferential labeling of the contralateral anterior ION was not apparent in mutant mice when Fluorogold was injected into the cerebellar hemisphere. This loss of labeling specificity was likely due to the death of the majority of the neurons in the anterior ION and the ectopic midbrain position of many Purkinje cells that should reside in the lateral cerebellum.

Together, our data demonstrate that unlike Ntn1 or Dcc, $U n c 5 c$ is not necessary for ventral migration of IO neurons or for guidance of IO axons across midline. However, this receptor is clearly necessary for continued dorsal guidance of IO axons away from midline. Interestingly, while some mutant IO neurons undergo apoptosis, our data demonstrate that many misrouted IO axons in the $U n c 5 c^{-1-}$ brain do ultimately reach Purkinje cells with correct mediolateral topography, presumably via utilization of other guidance cues.

\section{Disruption of cuneocerebellar guidance in the \\ Unc5 $c^{-/-}$hindbrain}

Our retrograde DiI tracing experiments demonstrated that the cuneocerebellar tract (CCT) of the $U n c 5 c^{-1-}$ ECN did not reach the cerebellum by $\mathrm{P} 0$, suggesting that the $\mathrm{ECN}$ and/or its axons develop abnormally in the mutant hindbrain (Fig. 3). ECN neurons are generated from the caudal rhombic lip between E13 and E15 and migrate tangentially toward the ventral midline. These neurons then cross the midline and continue migrating dorsally to populate the dorsal medulla. To determine whether ECN neurons properly migrate during development of this nucleus, we examined the developing ECN in wild-type and $U n c 5 c^{-1-}$ hindbrains at E14.5 during their migration and at E16.5 after their migration is completed. Atoh1 is expressed strongly in the posterior migration stream that includes migrating ECN and LRN precursors (Wang et al., 2005). Thus we generated $U n c 5 c^{-l-}$ embryos that were heterozygous for a lacZ replacement allele of Atoh1 (Atoh $1^{\text {lac } Z}$ ), in which lacZ expression mimics endogenous Atoh1 expression. X-Gal staining in the developing hindbrain of E14.5 Atoh1 $1^{\text {lacZ/+ }}$ and Unc5c $c^{-1-}$;Atoh $1^{\text {lacZ/+ }}$ embryos demonstrated similar patterns of staining at the ventral midline, suggesting that the ECN and LRN normally migrate in $U n c 5 c^{-1-}$ embryos (Fig. $6 A, B)$ ( $n=3$ for each genotype). At E16.5, the ECN was assessed by immunofluorescence with an antibody against BARHL1, which is highly expressed in the developing ECN. The structure of the ECN was similar in both the mutant and wild-type hindbrain, although the mutant nucleus was slightly elongated in a ventral direction compared to that of wildtype embryos (Fig. $6 C, D)$ ( $n=3$ for each genotype). In addition, the number of BARHL1-positive neurons was not significantly different between wild-type and $U n c 5 c^{-l-}$ hindbrains (wild type, $379 \pm 57$; mutant, $418 \pm 34$; mean $\pm \mathrm{SD}, p=0.65, n=3$ for each genotype; see Materials and Methods).

To determine the trajectories of the CCT in mutant mice, we placed DiI in the dorsolateral aspect of the medulla just under the caudal cerebellum, near to the ECN. In the wild-type brain the CCT and the dorsal spinotrigeminal tract ( $\mathrm{sp} 5$ ), which carries sensory information to the spinal trigeminal nucleus, were labeled (Fig. $6 E$ ). No labeled tracts projecting toward the cerebellum were observed in the $U n c 5 c^{-1-}$ brain when DiI was similarly placed (Fig. $6 F$ ). In addition, the dorsal sp5 tract appeared defasciculated in the mutant brainstem ( $n=3$ for each genotype). Interestingly, this analysis revealed an additional labeled tract 
WT
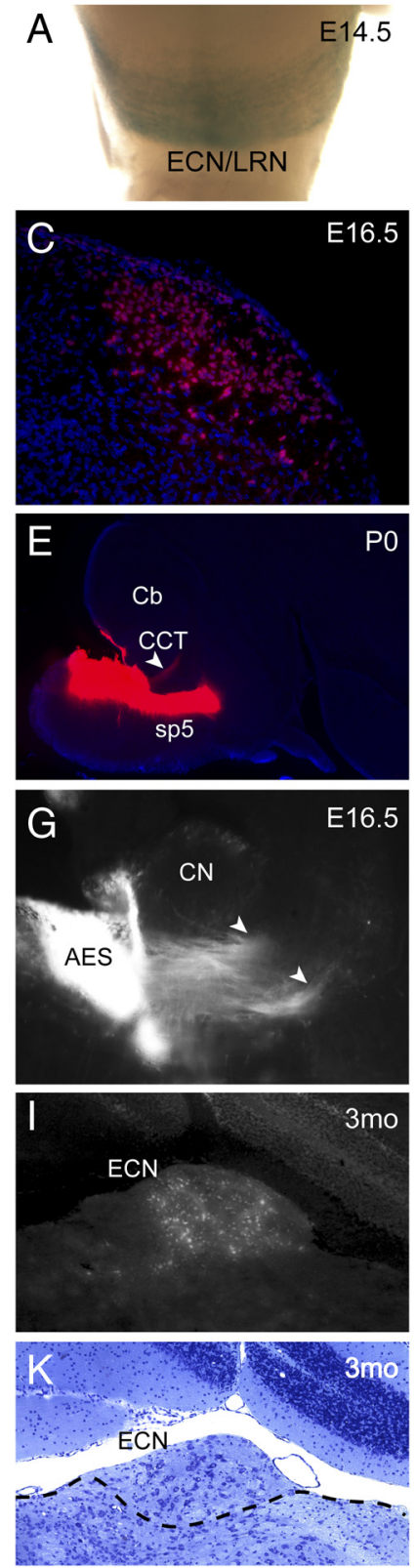

Unc5c
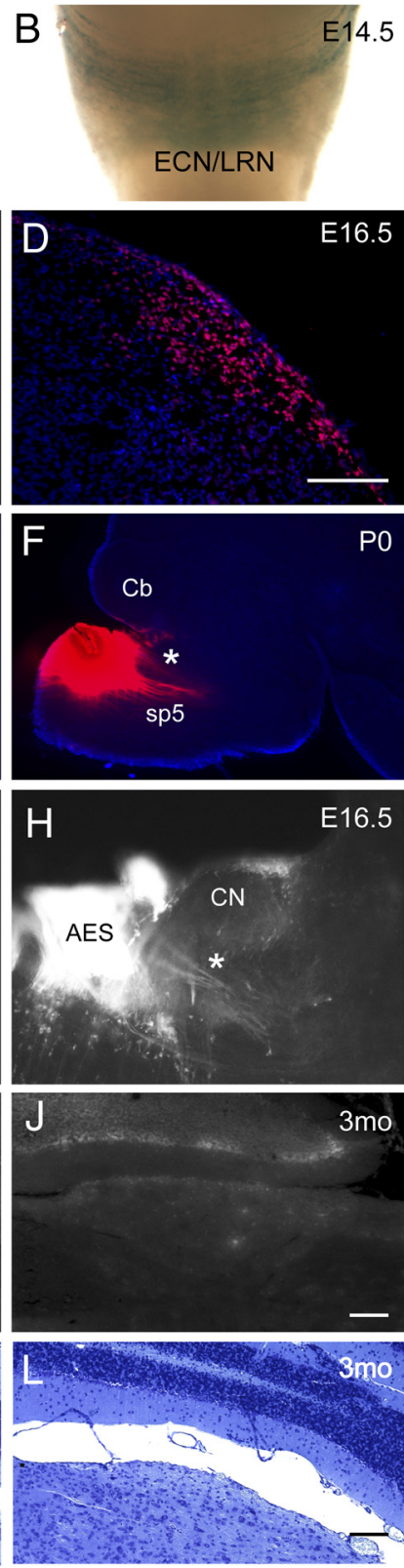

Figure 6. Axonal guidance defects and degeneration of neurons in external cuneate nucleus in

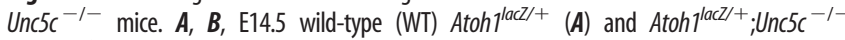
$\left(U n c 5 \mathrm{C}^{-I-}\right)(\boldsymbol{B})$ hindbrains were stained with X-Gal. The posterior migration stream containing migrating ECN/LRN neurons is shown in wholemount images of ventral medulla. $\boldsymbol{C}, \boldsymbol{D}$, Representative coronal sections of E16.5 wild-type $(\boldsymbol{C})$ and $U n \mathrm{CC}^{-1-}(\boldsymbol{D})$ hindbrains were immunostained with BARHL1 antibodies and counterstained with Hoechst dye. No significant differences in the number of immunoreactive cells in the ECN were observed between wild-type and mutant mice. $\boldsymbol{E}, \boldsymbol{F}$, A single Dil crystal was placed into the presumptive ECN region in the dorsal medulla of PO wild-type $(\boldsymbol{E})$ and UnC5c $\mathrm{C}^{-1-}(\boldsymbol{F})$ hindbrains. Sagittal sections were obtained $2 \mathrm{~d}$ after labeling and counter stained with Hoechst dye. Note the absence of the rostrodorsal projection of the presumptive CCT (arrowhead) in the mutant brainstem. Instead, an aberrant rostroventral projection (asterisk) is observed in the mutant brainstem. G, $\boldsymbol{H}$, Sagittal sections of the hindbrain of E16.5 Atoh1-tauGFP (WT) (G) and Atoh1tauGFP;UnC5C $\mathrm{C}^{-1-}\left(\right.$ UnC5C $\left.^{-1-}\right)(\boldsymbol{H})$ embryos. In the Atoh1-tauGFP hindbrain, the GFP-expressing fibers of the inferior cerebellar peduncle (arrowheads) extended in a dorsal direction from the ECN while in the Atoh1-tauGFP; UnC5 $\mathrm{C}^{-1-}$ hindbrain GFP-positive fibers (asterisk) extending from the ECN are ventrally directed. $I, J$, Fluorogold was injected into the cerebellum of 3-month-old (3mo) wild-type $(\boldsymbol{I})$ and $U n C 5 c^{-I-}(\boldsymbol{J})$ mice. The ECN was retrogradely labeled in the dorsal medulla of wild-type mice, while no labeling was observed in the mutant medulla. $\boldsymbol{K}, \boldsymbol{L}$, Representative cresyl violet-stained coronal sections from 3-month-old wild-type $(\boldsymbol{K})$ and $U n \mathrm{CC}^{-1-}(\boldsymbol{L})$ mice demonstrate the absence of the ECN (denoted by dashed lines in $\boldsymbol{K}$ ) in the Unc $5 \mathrm{C}^{-1-}$ brainstem. AES, Anterior extramural stream; Cb, cerebellum; CN, cochlear nucleus. Scale bars, $10 \mu \mathrm{m}$. that appeared to follow a rostroventral trajectory distinct from that of the dorsal sp5 tract. To confirm that this ectopic tract contains ECN axons, we used a reporter mouse in which axons and soma of Atoh1-expressing neurons can be identified by expression of a tauGFP fusion protein (Imondi et al., 2007). As expected, analysis of Atoh1-tauGFP hemizygous embryos demonstrated GFP-expressing cell bodies and axons of rhombic lip derivatives, including cerebellar granule cells, pontine neurons, and ECN/LRN neurons and the ICP (data not shown). We generated Atoh1-tauGFP;Unc5 $c^{-1-}$ embryos by crossing $U n c 5 c^{+1-}$ and Atoh1-tauGFP;Unc5c ${ }^{+/-}$mice. In the dorsal medulla of E16.5 Atoh1-tauGFP embryos, the fibers extending from ECN joined the ICP comprised of many GFP-positive fibers, which extended dorsally to the lateral cerebellum (Fig. 6G) (data not shown). However, the ICP was not present in this region of the mutant medulla, allowing clear visualization of the GFP-positive fibers extending from the ECN. Rather than turning dorsally toward the cerebellum, ECN fibers projected in a ventral direction (Fig. $6 H$ ). Together, these data suggest that dorsal migration of the CCT is abnormal in the $U n c 5 c^{-1-}$ brain.

To evaluate whether misguided ECN axons in the $U n c 5 c^{-1-}$ brain eventually reach the cerebellum as observed for axons of the olivocerebellar tract, we analyzed the dorsal medulla from adult wild-type and mutant mice after Fluorogold injection into the cerebellum. In wild-type brains, a strongly labeled cell cluster was detected in the dorsal medulla, which formed a small protuberance under the caudal cerebellum typical of the ECN (Fig. 6I). In mutant brains we did not detect labeled cells within the dorsal medulla, nor did we observe the protruding structure of the ECN or large diameter neurons by cresyl violet staining (Fig. 6J-L).

To determine whether ECN neurons, like the IO neurons, undergo apoptosis, we immunostained adjacent medullary sections from P0 wild-type and mutant mice with antibodies to cleaved caspase-3 and BARHL1 (supplemental Fig. 5G-J). Cleaved caspase-3-positive cells were observed in the mutant but not the wild-type ECN. These results demonstrate that many ECN neurons die after formation of the ECN in the $U n c 5 c^{-1-}$ hindbrain, perhaps due to the failure of these neurons to form synapses with cerebellar granule cell dendrites.

\section{Axon guidance and migration defects in pontine neurons}

Like the IO neurons, $U n c 5 c$ is expressed in the pontine neurons after they complete their migrations (Fig. 2C). To determine whether $U n c 5 c$ regulates $\mathrm{PN}$ axon guidance, we analyzed these axons in Atoh1-tauGFP;Unc5c $c^{-1-}$ embryos. Pontine neurons arise from the lower rhombic lip between E14 and E17 and tangentially migrate through the anterior extramural stream to the midline of the ventral pons. Axons of these neurons cross midline and then project dorsally toward the cerebellum. At E16.5, migrating pontine neurons and their axons strongly expressed GFP. In control embryos $(n=15)$, GFP-positive neurons were observed in the anterior extramural stream and at the position of the pontine nuclei, and their axons laterally projected toward the developing cerebellum (Fig. 7A) (Yee et al., 1999).

In contrast, the laterally projecting pontine axon tract was absent in Atoh1-tauGFP;Unc5 $c^{-1-}$ embryos $(n=9)$ (Fig. $\left.7 B\right)$. To directly visualize guidance of these axons, we placed DiI on the GFP-labeled pontine neurons of wild-type and mutant embryos (Fig. 7C,D). DiI-labeled axons in wild-type embryos crossed midline and continued migrating circumferentially $(n=5)$. In the mutant hindbrain, pontine axons also crossed midline but then turned rostrally $(n=9)$. In two of nine mutant embryos we observed both rostrally and caudally turning pontine axons (see 

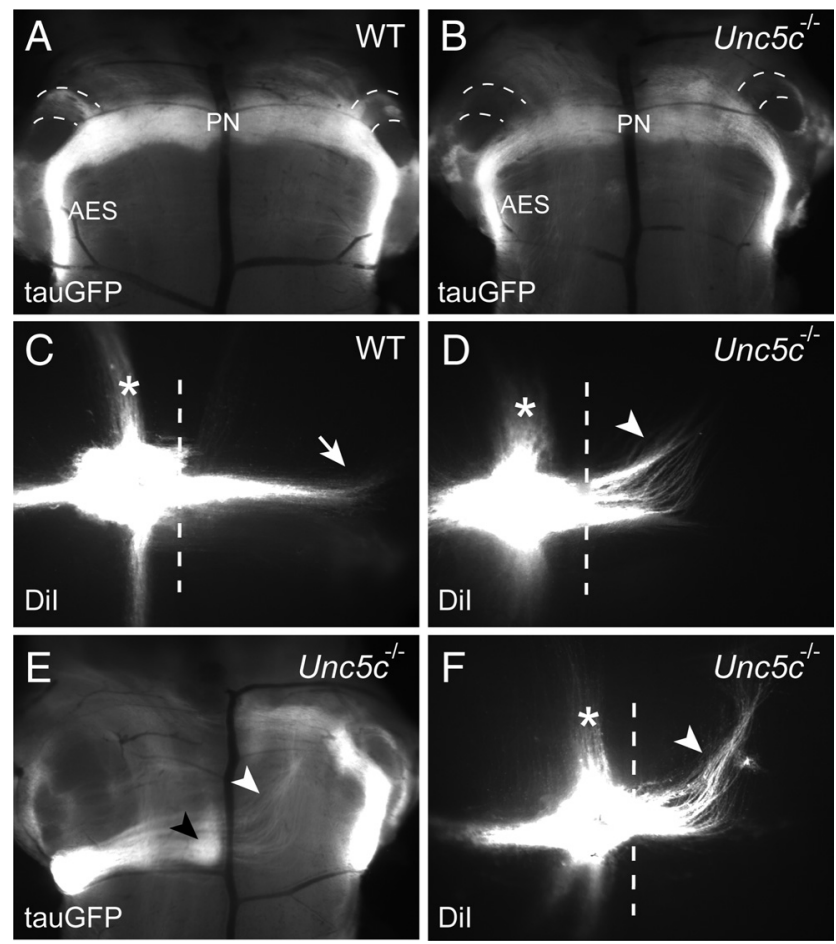

Figure 7. Abnormal migration and axonal guidance of $U n C 5 \mathrm{C}^{-1-}$ pontine neurons. $\boldsymbol{A}-\boldsymbol{F}$, Pontine neurons and their axons were examined in E16.5 wild-type (WT) Atoh1-tauGFP $(\boldsymbol{A}, \boldsymbol{C})$ or Atoh1-tauGFP;Unc5 $\mathrm{C}^{-1-}\left(\right.$ Unc5 $\left.^{-1-}\right)(\boldsymbol{B}, \boldsymbol{D}, \boldsymbol{E}, \boldsymbol{F})$ embryos. GFP $(\boldsymbol{A}, \boldsymbol{B}, \boldsymbol{E})$ or Dil-fluorescence $(\boldsymbol{C}, \boldsymbol{D}, \boldsymbol{F})$ is shown in wholemount images of the ventral hindbrain. $\boldsymbol{A}, \boldsymbol{B}, \mathrm{A}$ GFP-labeled pontine axon tract (flanked by dashed lines) projecting to the developing cerebellum is observed in the wild-type $(\boldsymbol{A})$, but not in the mutant $(\boldsymbol{B})$ hindbrain. $\boldsymbol{C}, \boldsymbol{D}$, Dil was placed into the pontine nuclei of Atoh 1-tauGFP (C) or Atoh1-tauGFP; UnC5 ${ }^{-1-}(\boldsymbol{D})$ embryos to directly detect the trajectories of pontine axons. In wild-type embryos, pontine axons projected laterally around the circumference of the hindbrain (arrow), while in mutant hindbrain these axons turned longitudinally after crossing midline (arrowhead). Rostrally turning axons are shown, but caudally turning axons were also observed in some mutant embryos. The position of the ventral midline is indicated by a dashed vertical line. Asterisks indicate a non-pontine Dil-labeled tract observed in

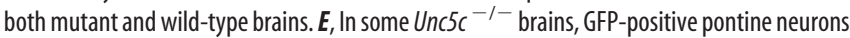
(black arrowhead) and their migration stream were ectopically located. A white arrowhead indicates GFP-positive axons projecting rostrally from ectopic pontine neurons. $\boldsymbol{F}$, Dil was placed in the ectopic nuclei shown in $\boldsymbol{E}$. Dil-labeled axons (arrowhead) also projected rostrally after crossing the midline.

Fig. 90). To address whether mutant axons reach the cerebellum, we unilaterally injected Fluorogold into the cerebellum of mutant and wild-type mice ( $n=3$ for each genotype). The contralateral pontine nucleus was labeled in both mice of both genotypes, demonstrating that although pontine axons require $U n c 5 c$ for continued circumferential dorsal guidance away from midline, these axons use an alternate route to reach the contralateral cerebellum (data not shown).

Unexpectedly, four of nine $U n c 5 c^{-l-}$ embryos analyzed had a dramatic reduction in the number of GFP-positive cells in the PN. However, these mice had ectopically located GFP-positive cells in the ventral midline of the medulla, midway between the normal location of the PN and the ION (Fig. 7E). Similar defects in $\mathrm{PN}$ formation were observed in $U n c 5 c^{-1-}$ embryos on an inbred C57BL/6J background when the PNs were visualized via introduction of the Atoh $1^{\text {lac } Z}$ gene (see Fig. 9). DiI labeling of axons projecting from these ectopic neurons demonstrated they followed a similar trajectory to that of normally positioned pontine neurons in the mutant brainstem, i.e., they crossed midline and then turned rostrally to project longitudinally toward the pons $(n=3)$ (Fig. $7 F)$.
Axon guidance of the DCN is disrupted in $U n c 5 c^{-1-}$ mice Histological analysis of adult $U n c 5 c^{-1-}$ mice demonstrated a dramatic reduction of the cerebellar commissure (Fig. 1C,D). This major output tract of the cerebellum forms by E14.5 and is largely composed of axons known as the hook bundle that project from the medial deep neurons to several nuclei in the contralateral brainstem, including the vestibular nuclei (Ito, 1984). The failure of the hook bundle to cross midline in the mutant cerebellum was confirmed by DiI labeling studies of E16.5 cerebella. Fibers of the decussating presumptive hook bundle and the contralateral DCN were labeled in wild-type but not $U n c 5 c^{-1-}$ cerebella (Fig. $8 A, B$ ). Failure of these axons to cross midline in the mutant cerebellum was also confirmed by immunostaining of E16.5 brain sections with antibodies to neurofilament $(165 \mathrm{kDa})$ (Fig. 8C,D).

To exclude the possibility that the failure of retrograde labeling of medial deep neurons in the mutant cerebellum comes from the malformation of the medial deep nucleus during development, we examined these neurons in E16.5 wild-type and $U n c 5 c^{-1-}$ cerebella. Medial deep neurons were visualized by immunostaining cerebellar sections with antibodies against TBR1, which is specifically expressed in this subset of deep neurons at this stage of development (Fig. 8E,F) (Fink et al., 2006). No significant difference was observed in TBR1-positive neurons between wild-type and mutant embryos (wild type, $707 \pm 124$; mutant, $525 \pm 93$, mean $\pm \mathrm{SD}, p=0.37 ; n=3$ for each genotype; See Material and Methods). Consistent with the rostral malformation of the cerebellum in $U n c 5 c^{-1-}$ mice and our histological analysis of adult mutant mice, TBR1-positive neurons were slightly displaced rostrally toward the caudal midbrain in Unc $5 c^{-1-}$ embryos (Fig. 1D) (data not shown).

To determine the path of mutant DCN axons, we unilaterally placed DiI in the cerebellum of wild-type and $U n c 5 c^{-1-}$ P0 mice ( $n=3$ for each genotype). The ICP and the descending and ascending SCPs were strongly labeled in wild-type mice as evidenced by an examination of sagittal sections from labeled brains (Fig. 8G) (data not shown). Consistent with our results described above, labeling of the ICP was severely reduced in $U n c 5 c^{-1-}$ neonates. However, labeling of the SCP in the mutant brain was similar to that observed in wild-type brains (Fig. 8G,H) (data not shown). Interestingly, an additional tract located dorsal to the SCP and projecting to the rostral midbrain was observed in $U n c 5 c^{-1-}$ neonates (Fig. $8 H$ ). To confirm that this ectopic tract was the misprojecting hook bundle, we immunostained the sections with antibodies against calretinin, which is expressed in the developing hook bundle (Fig. 8I,J) (Fink et al., 2006). Positive fibers projecting from the cerebellum toward the midbrain that resemble the projections of the DiI-labeled tract were observed in sections of the mutant brain (Fig. $8 \mathrm{H}$ ). Immunopositive axons were not observed in sections from the same level of the wild-type brain.

Further support for a role of $U n c 5 c$ in hook bundle development was obtained from an analysis of adult cerebella. Immunostaining of brain sections with a neurofilament antibody consistently labeled an abnormal axonal tract rostrally projecting from the cerebellum to the midbrain in $U n c 5 c^{-1-}$ but not wildtype brains, demonstrating that this ectopic tract was also maintained in the adult mutant mice (supplemental Fig. $7 A, B$, available at www.jneurosci.org as supplemental material). Injection of fluorescein-conjugated dextran amine, an anterograde neurotracer, into the medial/intermediate deep neurons of wildtype and mutant cerebella supported that this ectopic tract is indeed a cerebellar output tract (supplemental Fig. 7C,D). Together, our data suggest that axons of the medial deep cerebellar 

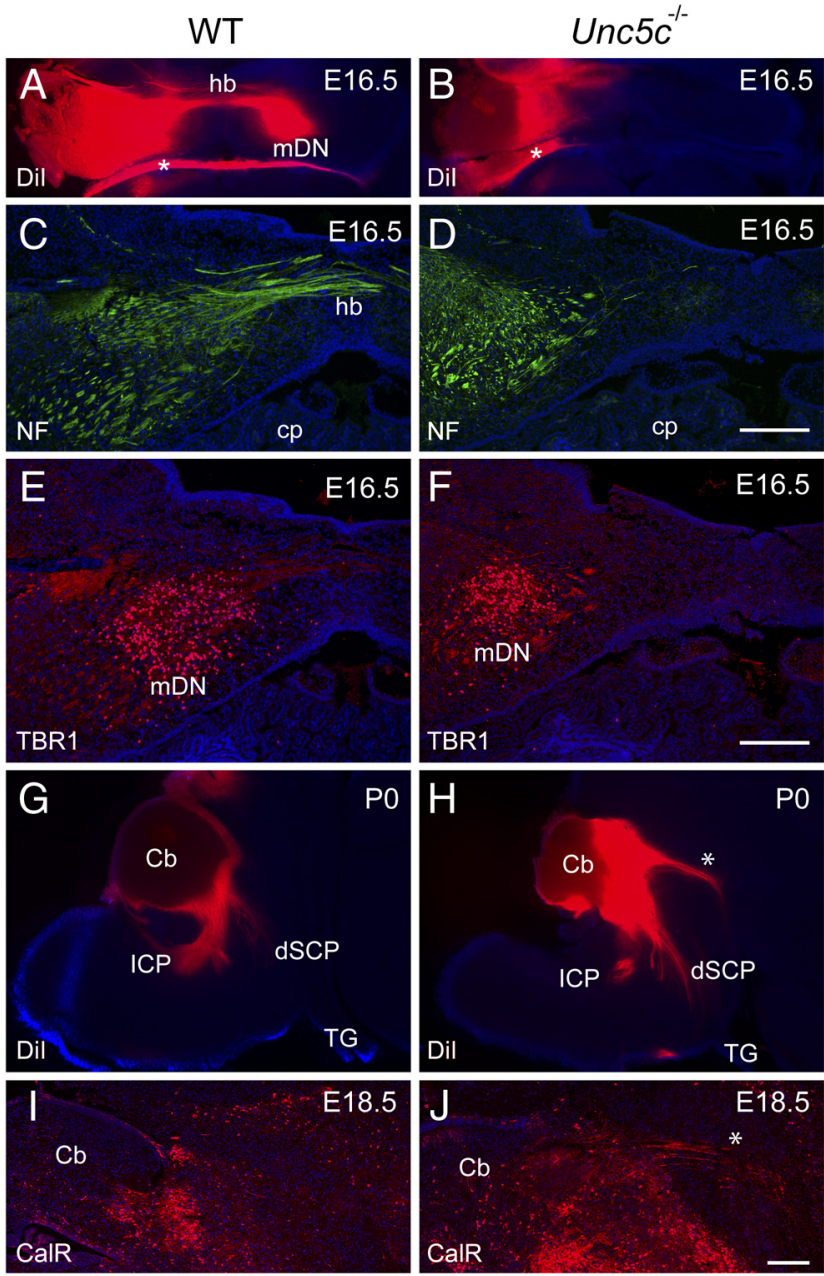

Figure 8. The dorsal guidance of axons from medial deep cerebellar neurons is disrupted in

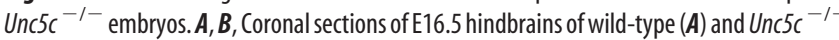
$(\boldsymbol{B})$ embryos unilaterally labeled with Dil in the cerebellar hemisphere. Note the absence of labeling of contralateral medial deep nucleus ( $\mathrm{mDN}$ ) and hook bundle (hb) in Unc5 $\mathrm{c}^{-1-} \mathrm{em}$ bryos. An asterisk indicates meninges-labeling artifacts. $C, D$, The absence of the hook bundle in the Unc5C $\mathrm{C}^{-1-}$ cerebellum was confirmed in coronal sections from E16.5 wild-type $(\boldsymbol{C})$ and Unc5c $\mathrm{C}^{-1-}(\boldsymbol{D})$ cerebella immunostained with antibody against neurofilament (NF; $165 \mathrm{kDa}$ ). $\boldsymbol{E}$, $\boldsymbol{F}$, Coronal sections from E16.5 wild-type $(\boldsymbol{E})$ and $U n C 5 c^{-1-}(\boldsymbol{F})$ embryos were immunostained with antibody against TBR1. Representative sections are shown. The number of TBR1-positive deep neurons in the Unc $5 \mathrm{C}^{-1-}$ hindbrain was not different from that of wild-type mice. $\boldsymbol{G}, \boldsymbol{H}$, Sagittal sections of the Dil-labeled brainstem from wild-type $(\boldsymbol{G})$ and $U n \mathrm{CC}^{-/-}(\boldsymbol{H})$ embryos. Note the presence of an abnormal tract (denoted by an asterisk) projecting rostrally from the cerebellum in UnC5C $\mathrm{C}^{-I-}$ embryos. $\boldsymbol{I}, \boldsymbol{J}$, Sagittal sections of brains from E18.5 wild-type $(\boldsymbol{I})$ and Unc5 $\mathrm{C}^{-1-}(\boldsymbol{J})$ embryos were immunostained by antibody against calretinin, expressed by axons of the hook bundle. An ectopic calretinin-positive tract (asterisk) resembling the trajec-

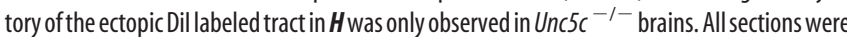
counterstained by Hoechst dye. Cb, Cerebellum; $C p$, choroid plexus; dSCP, descending superior cerebellar peduncle; TG, trigeminal nerve. Scale bars, $10 \mu \mathrm{m}(\boldsymbol{C}-\boldsymbol{F}, \boldsymbol{I}, \boldsymbol{J})$.

neurons in $U n c 5 c^{-1-}$ mice fail to decussate in the cerebellum and instead project to the ipsilateral rostral midbrain.

Transgenic expression of $U n c 5 c$ rescues pontine nucleus and hook bundle development but not cuneocerebellar tract guidance

During the development of the hindbrain, $U n c 5 c$ is expressed both in the NTZ and the posterior migratory stream in the medulla that gives rise to neurons of the ECN and the LRN. The transcription factor Atohl is expressed in many rhombic lip derivatives, including the precursors of the developing deep nuclei and the ECN (Wang et al., 2005). To address whether Unc5c acts cell autonomously in controlling the axon guidance of deep neurons, ECN neurons, and pontine neurons, we generated transgenic mice expressing an epitope-tagged $U n c 5 c$ cDNA under the control of the Atoh 1 promoter (supplemental Fig. $8 A$, available at www.jneurosci.org as supplemental material). This promoter has been shown to drive expression in a manner similar to endogenous Atoh1 (Helms et al., 2000). Northern analysis of RNA prepared from the P7 cerebellum of five independent transgenic lines demonstrated that the expression of the $U n c 5 c$ transgene was 2-10 times above that of the endogenous gene (data not shown).

We further analyzed two mouse lines that expressed the transgene at $\sim 5$ - and 10-fold levels over that of endogenous $U n c 5 c$. Immunohistochemistry using antibodies to the Myc epitope was performed to confirm correct expression of the transgene. Like endogenous Atoh1, the transgene was expressed in the NTZ and the external granule cell layer (EGL) at E14.5 (supplemental Fig. 8 B). Transgene expression was also evaluated by immunofluorescence with UNC5C antibodies in TgAtoh1-Unc5c;Unc5 $c^{-1-}$ embryos. As observed with anti-Myc antibodies, UNC5C transcripts were detected in the EGL and anterior migration stream as well as the rhombic lip in both lines (supplemental Fig. 8C). Although expression of $U n c 5 c$ in the wild-type ECN is not detectable at E16.5, $U n c 5 c$ was clearly detectable in the ECN of TgAtoh $1-U n c 5 c$ at this time (supplemental Fig. $8 D-G$ ). No abnormalities were observed in precerebellar or cerebellar structures in TgAtoh1-Unc5c mice, nor were obvious behavioral defects observed (data not shown).

We have previously demonstrated that $U n c 5 c^{-1-}$ mice have ectopic migration of embryonic granule cell and Purkinje cell progenitors into the lateral pons and the inferior colliculus (Ackerman et al., 1997; Przyborski et al., 1998). Analysis of aggregation chimeras demonstrated that $U n c 5 c$ functions in migrating granule cell progenitors to define the anterior boundary of the cerebellum rather than in Purkinje cell progenitors, which comigrate to ectopic positions (Goldowitz et al., 2000). To determine whether the expression of $U n c 5 c$ in Atoh1-positive granule neuron progenitors can rescue the mismigration of $U n c 5 c^{-1-}$ progenitors, we examined the cerebellum and midbrain of mutant mice expressing the Atoh1-Unc5c transgene (supplemental Fig. 9, available at www.jneurosci.org as supplemental material). Expression of Unc5c in Atoh1-expressing granule cell progenitors in either transgenic line partially rescued the midbrain ectopias in $U n c 5 c^{-1-}$ mice and largely restored the normal size of rostral vermis (supplemental Fig. 9) (data not shown), confirming functional expression of the Atoh1-Unc5c transgene.

To determine whether $U n c 5 c$ expression in deep neurons is sufficient to rescue hook bundle guidance, we placed DiI crystals unilaterally into the cerebellum of E16.5 TgAtoh1-Unc5c and TgAtoh1-Unc5c;Unc5c $c^{-1-}$ mice. As observed in wild type, DiI labeled the contralateral medial deep neurons via the hook bundle in TgAtoh1-Unc5c brains (Fig. 9A,B) $(n=3)$. We also observed that the labeling of the contralateral medial deep neurons and hook bundle in TgAtoh1-Unc5c;Unc5c $c^{-1-}$ mice, generated by crosses to either TgAtoh1-Unc5c transgenic line, was similar to that observed in the wild-type brain, demonstrating that transgenic $U n c 5 c$ expression in the medial deep neurons rescues this projection in $U n c 5 c^{-1-}$ embryos (Fig. $\left.9 C, D\right)(n=3)$. Rescue of hook bundle guidance in TgAtoh1-Unc5c;Unc5c-1- mice was also supported by the loss of the abnormal tract that projects to the rostral midbrain in $U n c 5 c^{-1-}$ mice (data not shown). Atoh1 is not expressed in the developing ION (Wang et al., 2005). In agreement, the olivocerebellar tract was not labeled by DiI in 

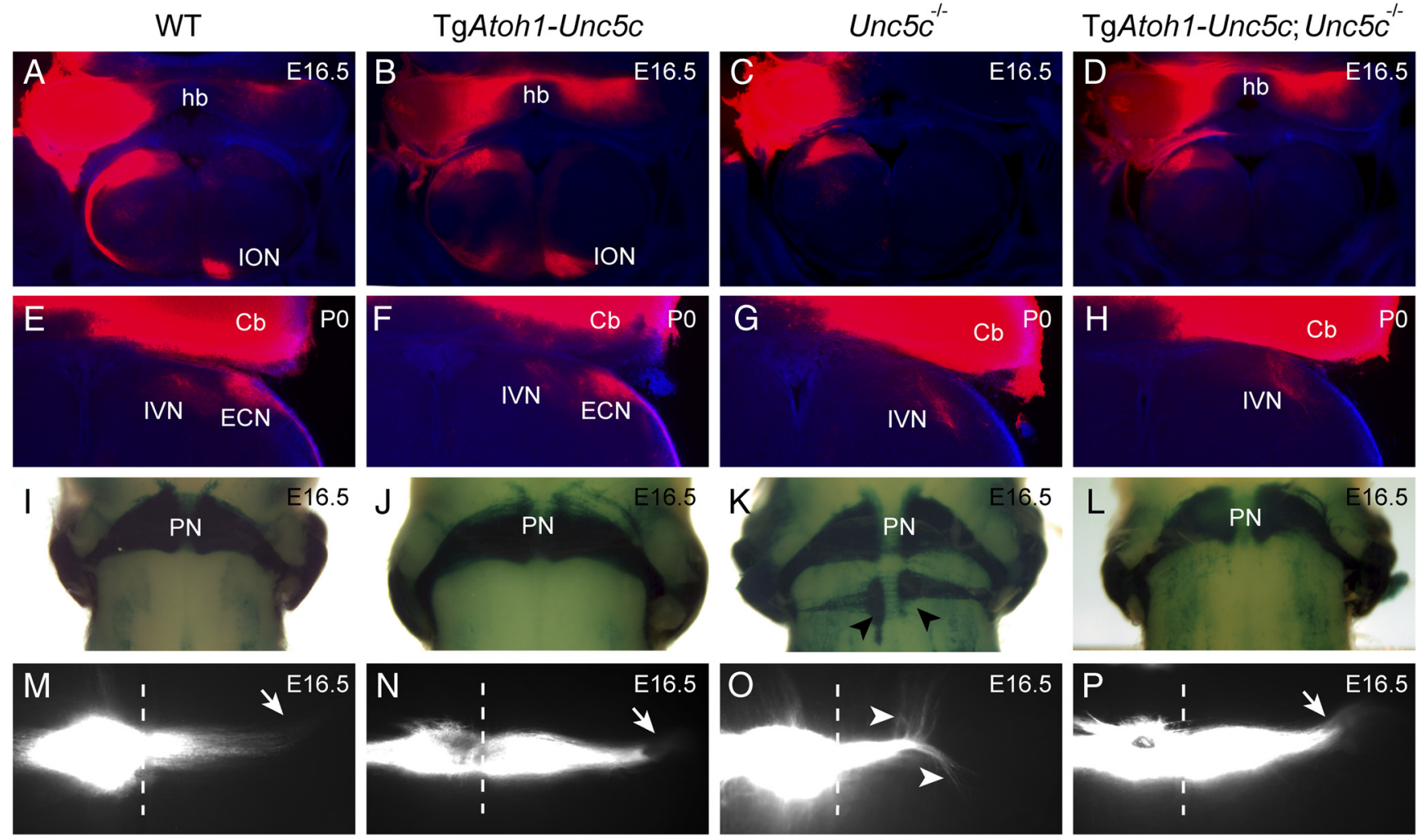

Figure 9. Unc $5 \mathrm{C}$ transgene expression in Atoh1-expressing neurons rescues the axonal guidance of the hook bundle and pontine migration but not the cuneocerebellar tract in Unc $5 \mathrm{C}^{-1-}$ mice.

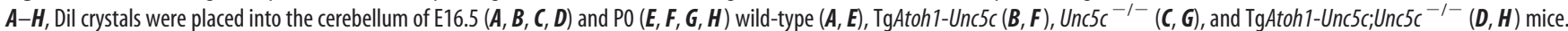
Hindbrains were sectioned coronally to visualize cerebellar tracts and counterstained with Hoechst dye. Note that transgenic expression of Unc 5 c rescues the cerebellar midline crossing of the hook bundle (hb), but not the axonal projections of the 10 neurons in TgAtoh1-Unc5c:Unc5 ${ }^{-1-}$ neonatal mice (D). Retrograde labeling of the ECN was not observed in TgAtoh1-Unc5c:Unc5 ${ }^{-1-}$ neonatal mice $(\boldsymbol{H})$. Note that the presumptive inferior vestibular tract (IVN) is similarly labeled in all of the mice. $\boldsymbol{I}-\boldsymbol{L}$, Pontine neuron migration was assessed by X-Gal staining of hindbrains from E16.5 embryos of each genotype that are also heterozygous for the Atoh $7^{\text {lacz/+ }}$ reporter gene. Arrowheads indicate ectopic formation of PN in mutant $(\boldsymbol{K})$. Transgenic expression of Unc5 $\mathrm{Crescues}$ ectopic formation of $\mathrm{PN}(\boldsymbol{L}) \cdot \boldsymbol{M}-\boldsymbol{P}$, Axon migration of pontine neurons was visualized by unilateral Dil insertion. Dil was inserted in the normally positioned pontine nucleus in $U n c 5 c^{-l-}$ brains $(\boldsymbol{O})$. Arrows indicate normal projections of pontine neurons while arrowheads indicate rostrally and caudally projecting axons in the Unc $5 \mathrm{c}^{-1-}$ hindbrain. Cb, Cerebellum.

Unc $5 c^{-1-}$ embryos expressing the Atoh1-Unc5c transgene from either line (Fig. 9D) $(n=3)$ at E16.5.

We assessed whether ECN axon guidance is directly regulated by Unc5c expression by placing DiI in the cerebellum of P0 TgAtoh1-Unc5c;Unc5c $c^{-1-}$ mice. DiI labeled the ECN located adjacent to the inferior vestibular tract in both wild-type and TgAtoh1Unc5c brains (Fig. 9E,F) ( $n=3$ for both genotypes). However, like $U n c 5 c^{-l-}$ brains, no labeling of the ECN was observed in $\operatorname{TgAtoh} 1-U n c 5 c ; U n c 5 c^{-1-}$ mice generated by crosses to either transgenic line, suggesting that Unc5c expression in the mutant ECN does not rescue the guidance of ECN axons (Fig. 9G,H) ( $n=3$ for both genotypes). This suggestion was confirmed by both Fluorogold injection into the cerebellum of adult TgAtoh1$U n c 5 c ; U n c 5 c^{-1-}$ mice and histological analysis of the ECN in these mice. These analyses demonstrated that degeneration of the ECN occurred in TgAtoh1-Unc5c;Unc5 $c^{-1-}$ mice as observed in $U n c 5 c^{-1-}$ mice (supplemental Fig. 10, available at www.jneurosci.org as supplemental material).

Lastly, to ask whether transgenic expression of $U n c 5 c$ rescues pontine neuron phenotypes, we generated Atoh $1^{\text {lacZ/+ }} ;$ TgAtoh1Unc5c;Unc5 $c^{-1-}$ mice by crossing TgAtoh $1-U n c 5 c ; U n c 5 c^{+1-}$ and Atohl ${ }^{\text {lacZ/+ }} ; U_{n c 5 c^{+/-}}$mice. X-Gal staining revealed ectopic pontine neuron clusters at the ventral midline in five of seven Atoh $1^{\text {lacZl+ }} ; U n c 5 c^{-1-}$ embryos, but no ectopic pontine neurons were observed in Atoh $1^{\text {lacZ/+ }}(n=6)$, Atoh $1^{\text {lacZ/+ }} ;$ TgAtoh1-Unc5c $(n=3)$, or Atoh1 $1^{\text {lacZ/+ }} ; \operatorname{Unc} \mathrm{c}^{-1-}(n=4)$ embryos carrying the
TgAtoh1-Unc5c transgene (Fig. 9I-L). Pontine axon migration was assessed in TgAtoh1-Unc5c;Unc5c ${ }^{-1-}$ mice by direct DiI labeling of the pontine nucleus. Like wild-type axons, pontine axons in TgAtoh1-Unc5c;Unc5c ${ }^{-1-}$ mutant embryos $(n=3)$ migrated circumferentially in a lateral direction rather than longitudinally as observed in $U n c 5 c^{-1-}$ embryos and was indistinguishable from wild-type and $\operatorname{TgAtoh1-Unc5c}(n=4)$ controls (Fig. 9M-P). No defects in any of these structures were noted in $\operatorname{Tg}$ Atoh1-Unc $5 c$ mice from either line.

Together, our results suggest that $U n c 5 c$ functions in a cellautonomous manner in the guidance of axons of medial deep neurons and pontine neurons and in pontine neuron migration. However, transgenic expression of Unc5c in the developing ECN did not rescue guidance of the cuneocerebellar tract, raising the possibility that guidance defects in this tract may not be due to the loss of Unc5c expression in ECN neurons.

\section{Discussion}

$U n c 5 c$ regulates the long-range dorsal guidance of inferior olivary and pontine neurons

In contrast to our knowledge of molecules regulating ventral attraction of migrating axons and neurons, the guidance cues necessary for dorsal projections of axons, including the IO and PN axons, away from the midline are largely unknown. Unlike $N t n 1^{-1-}$ or $D c^{-1-}$ embryos, which have defects in the ventral attraction of IO and PN axons to midline, both ventral migration 

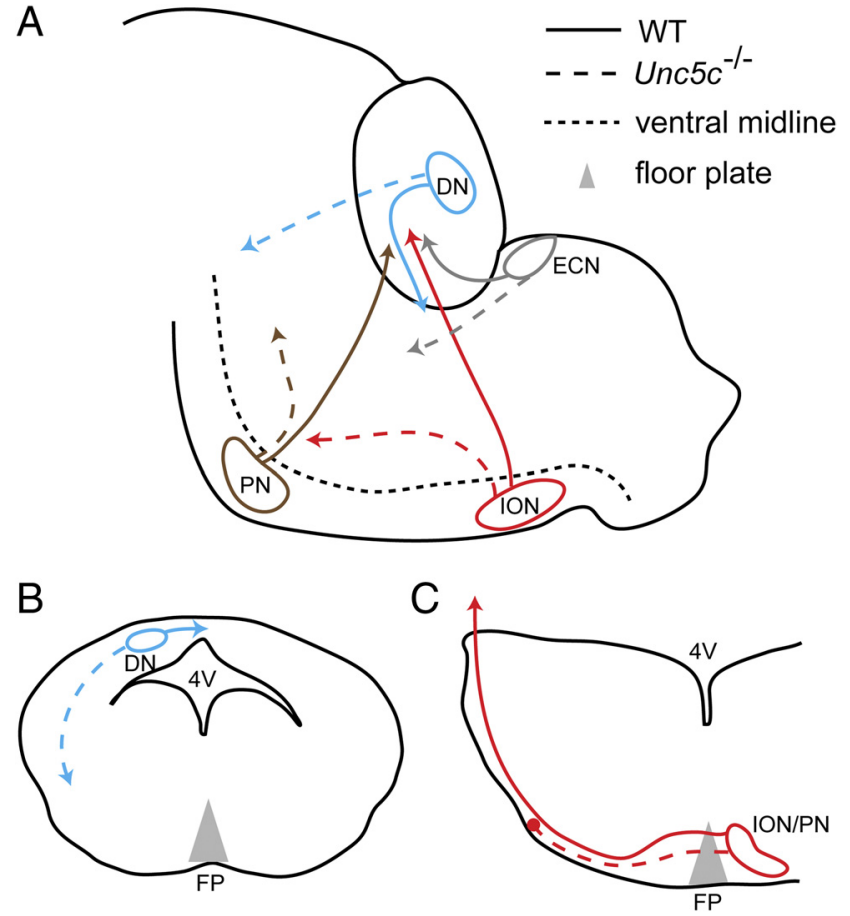

Figure 10. Precerebellar and cerebellar axon guidance defects in the $U n C 5 \mathrm{C}^{-1-}$ hindbrain. $A$, The hook bundle (blue), olivocerebellar tract (red), cuneocerebellar tract (gray), and pontocerebellar tract (brown) normally project dorsally away from the ventral midline of the hindbrain (solid lines). However, in Unc $5 \mathrm{C}^{-1-}$ mice these tracts abnormally project in a ventral direction toward the pons (dashed lines). $\boldsymbol{B}, \boldsymbol{C}$, The proposed role of Unc 5 in the guidance of hook bundle and olivocerebellar/pontocerebellar tract at E13.5. B. UnC5c mediates the initial dorsal guidance of the hook bundle (solid line) as it projects contralaterally in the cerebellum, as illustrated by the schematic of a horizontal section of hindbrain through the developing cerebellum at E13.5. Loss of Unc5c blocks the dorsal guidance of these axons, and instead an unknown cue(s) results in the projection of axons toward the floor plate (dashed line). C, Unc5c mediates long-range dorsal migration of olivocerebellar axons from the floor plate toward the cerebellum as illustrated by the schematic of a coronal section of developing medulla at E13.5. Loss of Unc5c results in stalling (red circle) of dorsal migration of these tracts (dashed line) as they migrate away from the floor plate. Instead, axons turn rostrally and eventually innervate the cerebellum. Pontine neurons shows similar dorsal migration defects, except that some of pontine axons are also directed toward caudal medulla (shown in Fig. 90).

of $U n c 5 c^{-1-}$ neurons to midline and midline crossing of mutant axons are normal (Yee et al., 1999; Marcos et al., 2009). However, $U n c 5 c^{-1-}$ axons do not dorsally navigate along the circumference of the brainstem. Instead, mutant axons abruptly turn rostrally (or caudally in some cases) after crossing the midline and extend laterally for a short distance. These axons then project longitudinally along the ventral hindbrain (Fig. 10). Although Unc5c-deficient IO and PN axons fail to use normal migration pathways, many but not all of these axons do eventually reach the cerebellum and likely form synapses with their targets. This finding suggests that at least some of these axons are able to respond to different guidance cues secreted by the guidepost cells they encounter along their new migration route.

Like $U n c 5 c$, the Slit receptor Robo also plays a role in the guidance of axons after crossing the midline. However, axonal guidance defects in $U n c 5 c^{-1-}$ embryos are different from those observed in Slit/Robo-deficient embryos, where cerebellofugal and spinal commissural axons often fail to leave midline (Long et al., 2004; Tamada et al., 2008; Jaworski et al., 2010). Thus, our results suggest that unlike the role of Robo in short-range repulsion, Unc5c controls long-range, dorsal guidance of IO and PN axons away from the midline.

\section{Unc5c regulation of $\mathrm{ECN}$ and $\mathrm{LRN}$ axons}

The cuneocerebellar and reticulocerebellar tracts both join the olivocerebellar and spinocerebellar tract to form the ICP, but do so at different points. The cuneocerebellar tract projects rostrally from the dorsal medulla and joins the olivocerebellar tract as it enters the cerebellum, whereas axons of the reticulocerebellar tract join the olivocerebellar tract in the lateral aspects of the olivocerebellar tract (Altman and Bayer, 1997). In contrast to mice with mutations in the netrin-1/DCC and Slit/Robo guidance system, we do not observe defects in the formation of ECN/ LRN in $U n c 5 c^{-1-}$ embryos, suggesting a specific role in axon guidance (Di Meglio et al., 2008; Marcos et al., 2009). The Unc $5 c^{-1-}$ cuneocerebellar axons do not turn dorsally toward, nor do they innervate, the cerebellum. In contrast, retrograde labeling demonstrates that the mutant reticulocerebellar tract projects to the cerebellum. However, in the $U n c 5 c^{-1-}$ hindbrain we do not observe labeling of the lateral tract where these fibers normally transverse, suggesting that like the IO axons, axons of the LRN may use an ectopic route to transverse the hindbrain to the cerebellum (Fig. 3). This remains to be tested in future studies.

Interestingly, although $U n c 5 c$ is clearly expressed in ECN of $U n c 5 c^{-1-}$ embryos that carry the Atoh1 promoter-driven Unc5c transgene, guidance of this tract was not rescued by $U n c 5 c$ expression. Although we cannot rule out subtle $U n c 5 c$ expression differences between wild-type and transgenic mice, this finding suggests that ECN axonal abnormalities in the $U n c 5 c^{-1-}$ hindbrain are non-cell-autonomous. The Unc5c transgene is not expressed in the ION. Thus. IO axons still use their abnormal rostral/ventral route in $U n c 5 c^{-1-}$ mice carrying the transgene, raising the suggestion that ECN may normally fasciculate on the olivocerebellar tract on its way to the developing cerebellum (Altman and Bayer, 1997).

\section{Cell death of IO and ECN neurons in Unc5c mutant mice}

Our results demonstrate that many IO and ECN neurons degenerate in the hindbrain of $U n c 5 c^{-1-}$ mice during the early postnatal period. Many IO neurons also degenerate in Ntn1-deficient embryos. However, cell death commences at a much earlier time point (E12.5), suggesting that the mechanism of cell death is likely different (Llambi et al., 2001). Climbing fibers begin making synaptic contacts with Purkinje cells at E19, close to the time when we observe apoptosis of IO neurons (Mason et al., 1990). Our data suggest that mutant axons are delayed in reaching the cerebellum, hence their death is consistent with target-dependent cell loss. Interestingly, we observe neuronal death specifically in the DAO and PO. No specific increase in cell death was reported for this subset of IO neurons in mouse models of early Purkinje cell degeneration or in models in which Purkinje cells fail to develop (Chu et al., 2000). This suggests that the specific cell death of DAO and PO neurons is unlikely to be due to increased sensitivity of these neurons to target deprivation.

IO neurons project their axons to specific zones of Purkinje cells along the mediolateral axis of the cerebellum (Azizi and Woodward, 1987). Climbing fibers of the DAO and PO regions, which are mostly located in the anterior portion of the ION, primarily project to Purkinje cells in the cerebellar hemispheres. Granule cells and Purkinje cells from the lateral regions of the $U n c 5 c^{-1-}$ cerebellum mismigrate to the midbrain and pons during cerebellar development (Ackerman et al., 1997). This mismigration is accompanied by disruption of the cytoarchitecture of the cerebellar hemispheres. Thus, if mutant climbing fibers from the anterior regions of the ION retain their topographic specificity (as observed in mutant climbing fibers from the posterior 
ION), it is likely many DAO and PO axons project to Purkinje cells located in highly disorganized hemispheres or to neurons ectopically located in the midbrain. It is possible that these climbing fibers do not make proper synaptic contacts and/or lack proper trophic support and degenerate. Although it is not clear whether mutant ECN axons reach the cerebellum, similar defects in synaptic development may also underlie degeneration of ECN neurons.

\section{Unc $5 c$ regulates anterior migration of pontine neurons}

Defects in the ventral migration of the PN were reported in $N t n 1^{-1-}$ and $D c c^{-1-}$ embryos (Serafini et al., 1996; Fazeli et al., 1997; Yee et al., 1999). In contrast, in Unc5c ${ }^{-l-}$ embryos the PN neurons fail to complete their anterior migration, but the ventral positioning of these neurons was unaffected. Transgenic expression of $U n c 5 c$ in pontine neurons rescued the migration defects, suggesting that $U n c 5 c$ acts cell autonomously in pontine migration. However, migration defects were variable and not fully penetrant in mutant embryos even on an inbred genetic background, suggesting that pontine migration may also be under the influence of stochastic events.

Defects in AP positioning of migrating pontine neurons have also been observed in Slit/Robo mutants, suggesting that Slit/ Robo signaling may be required to antagonize netrin-1/DCC attraction at the choice point between ventral and rostral migration (Geisen et al., 2008). Unc5c may also function in negative regulation of netrin-1/DCC-mediated attraction in migrating PN, thereby facilitating the Slit/Robo responsiveness of these neurons. Analysis of both the spatiotemporal expression of $U n c 5 c$ in migrating PN neurons and the precise nature of migration defects is needed to further understand the mechanism of $U n c 5 c$ in regulating $\mathrm{PN}$ migration.

\section{Unc $5 c$ regulates the initial dorsal guidance of medial deep neurons in developing cerebellum}

Our experiments demonstrate that $U n c 5 c^{-1-}$ mice have abnormal ipsilateral projections of the medial deep neurons with a concomitant reduction of the cerebellar commissure. Dorsally crossing axons, including the trochlear axons and posterior commissural axons, were repelled by the floor plate in explant studies (Shirasaki et al., 1995; Serafini et al., 1996). Direct labeling demonstrated that the Unc5c $\mathrm{c}^{-1-}$ hook bundle projects toward the ipsilateral mesencephalic floor plate. Thus, $U n c 5 c$ may play a role in repelling the hook bundle from the floor plate, a suggestion that is supported by rescue of this abnormal projection by transgenic expression of $U n c 5 c$ in deep neurons.

Ntn1 expression is high in the ventral midline of a developing neural tube, raising the possible role as a guidance cue for dorsally guided axons. However, Ntn1 alone is not sufficient for many dorsally guided axons, including the posterior commissure, trochlear axons, and the hook bundle, suggesting that other functional ligands for UNC5C exist (Serafini et al., 1996; BlochGallego et al., 1999; Burgess et al., 2006). For example, Caenorhabditis elegans UNC-5 has recently been shown to bind UNC-129, which in turn enhances the long-range repulsive activity of UNC-5 (MacNeil et al., 2009). UNC5C was also shown to bind the vertebrate homolog of UNC-129, BMP7 (MacNeil et al., 2009). BMP7, which is expressed in the dorsal neural tube, has been shown to regulate spinal commissural axon development (Butler and Dodd, 2003). Interestingly, the migration of medullary precerebellar neurons has been shown to depend on alar plate-mediated attraction as well as floor plate-mediated repulsion; however, it remains to be determined whether $B m p 7$, like
Unc5c, plays a role in hindbrain development (Taniguchi et al., 2002).

\section{References}

Ackerman SL, Kozak LP, Przyborski SA, Rund LA, Boyer BB, Knowles BB (1997) The mouse rostral cerebellar malformation gene encodes an UNC-5-like protein. Nature 386:838-842.

Altman J, and Bayer SA (1997) Development of the cerebellar system: In relation to its evolution, structure, and functions. Boca Raton, FL: CRC.

Altman J, Bayer SA (1985) Embryonic development of the rat cerebellum. II. Translocation and regional distribution of the deep neurons. J Comp Neurol 231:27-41.

Altman J, Bayer SA (1987a) Development of the precerebellar nuclei in the rat: II. The intramural olivary migratory stream and the neurogenetic organization of the inferior olive. J Comp Neurol 257:490-512.

Altman J, Bayer SA (1987b) Development of the precerebellar nuclei in the rat: III. The posterior precerebellar extramural migratory stream and the lateral reticular and external cuneate nuclei. J Comp Neurol 257:513-528.

Altman J, Bayer SA (1987c) Development of the precerebellar nuclei in the rat: IV. The anterior precerebellar extramural migratory stream and the nucleus reticularis tegmenti pontis and the basal pontine gray. J Comp Neurol 257:529-552.

Azizi SA, Woodward DJ (1987) Inferior olivary nuclear complex of the rat: morphology and comments on the principles of organization within the olivocerebellar system. J Comp Neurol 263:467-484.

Bloch-Gallego E, Ezan F, Tessier-Lavigne M, Sotelo C (1999) Floor plate and netrin-1 are involved in the migration and survival of inferior olivary neurons. J Neurosci 19:4407-4420.

Burgess RW, Jucius TJ, Ackerman SL (2006) Motor axon guidance of the mammalian trochlear and phrenic nerves: dependence on the netrin receptor Unc5c and modifier loci. J Neurosci 26:5756-5766.

Butler SJ, Dodd J (2003) A role for BMP heterodimers in roof platemediated repulsion of commissural axons. Neuron 38:389-401.

Causeret F, Danne F, Ezan F, Sotelo C, Bloch-Gallego E (2002) Slit antagonizes netrin-1 attractive effects during the migration of inferior olivary neurons. Dev Biol 246:429-440.

Chu T, Hullinger H, Schilling K, Oberdick J (2000) Spatial and temporal changes in natural and target deprivation-induced cell death in the mouse inferior olive. J Neurobiol 43:18-30.

de Diego I, Kyriakopoulou K, Karagogeos D, Wassef M (2002) Multiple influences on the migration of precerebellar neurons in the caudal medulla. Development 129:297-306.

Di Meglio T, Nguyen-Ba-Charvet KT, Tessier-Lavigne M, Sotelo C, Chédotal A (2008) Molecular mechanisms controlling midline crossing by precerebellar neurons. J Neurosci 28:6285-6294.

Fazeli A, Dickinson SL, Hermiston ML, Tighe RV, Steen RG, Small CG, Stoeckli ET, Keino-Masu K, Masu M, Rayburn H, Simons J, Bronson RT, Gordon JI, Tessier-Lavigne M, Weinberg RA (1997) Phenotype of mice lacking functional Deleted in colorectal cancer (Dcc) gene. Nature 386:796-804.

Fink AJ, Englund C, Daza RA, Pham D, Lau C, Nivison M, Kowalczyk T, Hevner RF (2006) Development of the deep cerebellar nuclei: transcription factors and cell migration from the rhombic lip. J Neurosci 26:3066-3076

Geisen MJ, Di Meglio T, Pasqualetti M, Ducret S, Brunet JF, Chedotal A, Rijli FM (2008) Hox paralog group 2 genes control the migration of mouse pontine neurons through slit-robo signaling. PLoS Biol 6:e142.

Goldowitz D, Hamre KM, Przyborski SA, Ackerman SL (2000) Granule cells and cerebellar boundaries: analysis of Unc5h3 mutant chimeras. J Neurosci 20:4129-4137.

Helms AW, Abney AL, Ben-Arie N, Zoghbi HY, Johnson JE (2000) Autoregulation and multiple enhancers control Math1 expression in the developing nervous system. Development 127:1185-1196.

Hioki H, Fujiyama F, Taki K, Tomioka R, Furuta T, Tamamaki N, Kaneko T (2003) Differential distribution of vesicular glutamate transporters in the rat cerebellar cortex. Neuroscience 117:1-6.

Hong K, Hinck L, Nishiyama M, Poo MM, Tessier-Lavigne M, Stein E (1999) A ligand-gated association between cytoplasmic domains of UNC5 and DCC family receptors converts netrin-induced growth cone attraction to repulsion. Cell 97:927-941.

Imondi R, Jevince AR, Helms AW, Johnson JE, Kaprielian Z (2007) Mis- 
expression of $\mathrm{L} 1$ on pre-crossing spinal commissural axons disrupts pathfinding at the ventral midline. Mol Cell Neurosci 36:462-471.

Ito M (1984) The cerebellum and neural control. New York: Raven.

Jaworski A, Long H, Tessier-Lavigne M (2010) Collaborative and specialized functions of Robol and Robo2 in spinal commissural axon guidance. J Neurosci 30:9445-9453.

Keleman K, Dickson BJ (2001) Short- and long-range repulsion by the Drosophila Unc5 netrin receptor. Neuron 32:605-617.

Kuramoto T, Kuwamura M, Serikawa T (2004) Rat neurological mutations cerebellar vermis defect and hobble are caused by mutations in the netrin-1 receptor gene Unc5h3. Brain Res Mol Brain Res 122:103-108.

Kuwamura M, Ishida A, Yamate J, Kato K, Kotani T, Sakuma S (1997) Chronological and immunohistochemical observations of cerebellar dysplasia and vermis defect in the hereditary cerebellar vermis defect (CVD) rat. Acta Neuropathol 94:549-556.

Llambi F, Causeret F, Bloch-Gallego E, Mehlen P (2001) Netrin-1 acts as a survival factor via its receptors UNC5H and DCC. EMBO J 20:2715-2722.

Long H, Sabatier C, Ma L, Plump A, Yuan W, Ornitz DM, Tamada A, Murakami F, Goodman CS, Tessier-Lavigne M (2004) Conserved roles for Slit and Robo proteins in midline commissural axon guidance. Neuron 42:213-223.

MacNeil LT, Hardy WR, Pawson T, Wrana JL, Culotti JG (2009) UNC-129 regulates the balance between UNC-40 dependent and independent UNC-5 signaling pathways. Nat Neurosci 12:150-155.

Marcos S, Backer S, Causeret F, Tessier-Lavigne M, Bloch-Gallego E (2009) Differential roles of Netrin-1 and its receptor DCC in inferior olivary neuron migration. Mol Cell Neurosci 41:429-439.

Marillat V, Sabatier C, Failli V, Matsunaga E, Sotelo C, Tessier-Lavigne M, Chédotal A (2004) The slit receptor Rig-1/Robo3 controls midline crossing by hindbrain precerebellar neurons and axons. Neuron 43: 69-79.

Mason CA, Christakos S, Catalano SM (1990) Early climbing fiber interactions with Purkinje cells in the postnatal mouse cerebellum. J Comp Neurol 297:77-90.

Przyborski SA, Knowles BB, Ackerman SL (1998) Embryonic phenotype of Unc5h3 mutant mice suggests chemorepulsion during the formation of the rostral cerebellar boundary. Development 125:41-50.

Serafini T, Colamarino SA, Leonardo ED, Wang H, Beddington R, Skarnes
WC, Tessier-Lavigne M (1996) Netrin-1 is required for commissural axon guidance in the developing vertebrate nervous system. Cell 87:1001-1014.

Shirasaki R, Tamada A, Katsumata R, Murakami F (1995) Guidance of cerebellofugal axons in the rat embryo: directed growth toward the floor plate and subsequent elongation along the longitudinal axis. Neuron 14:961-972.

Shirasaki R, Mirzayan C, Tessier-Lavigne M, Murakami F (1996) Guidance of circumferentially growing axons by netrin-dependent and -independent floor plate chemotropism in the vertebrate brain. Neuron 17:1079-1088.

Sotelo C (2004) Cellular and genetic regulation of the development of the cerebellar system. Prog Neurobiol 72:295-339.

Tamada A, Kumada T, Zhu Y, Matsumoto T, Hatanaka Y, Muguruma K, Chen Z, Tanabe Y, Torigoe M, Yamauchi K, Oyama H, Nishida K, Murakami F (2008) Crucial roles of Robo proteins in midline crossing of cerebellofugal axons and lack of their up-regulation after midline crossing. Neural Dev 3:29.

Taniguchi H, Tamada A, Kennedy TE, Murakami F (2002) Crossing the ventral midline causes neurons to change their response to floor plate and alar plate attractive cues during transmedian migration. Dev Biol 249:321-332.

Wang VY, Rose MF, Zoghbi HY (2005) Math1 expression redefines the rhombic lip derivatives and reveals novel lineages within the brainstem and cerebellum. Neuron 48:31-43.

Wassef M, Chedotal A, Cholley B, Thomasset M, Heizmann CW, Sotelo C (1992) Development of the olivocerebellar projection in the rat: I. Transient biochemical compartmentation of the inferior olive. J Comp Neurol 323:519-536.

Welch JM, Wang D, Feng G (2004) Differential mRNA expression and protein localization of the SAP90/PSD-95-associated proteins (SAPAPs) in the nervous system of the mouse. J Comp Neurol 472:24-39.

Yamada M, Terao M, Terashima T, Fujiyama T, Kawaguchi Y, Nabeshima Y, Hoshino M (2007) Origin of climbing fiber neurons and their developmental dependence on Ptfla. J Neurosci 27:10924-10934.

Yee KT, Simon HH, Tessier-Lavigne M, O’Leary DM (1999) Extension of long leading processes and neuronal migration in the mammalian brain directed by the chemoattractant netrin-1. Neuron 24:607-622. 